
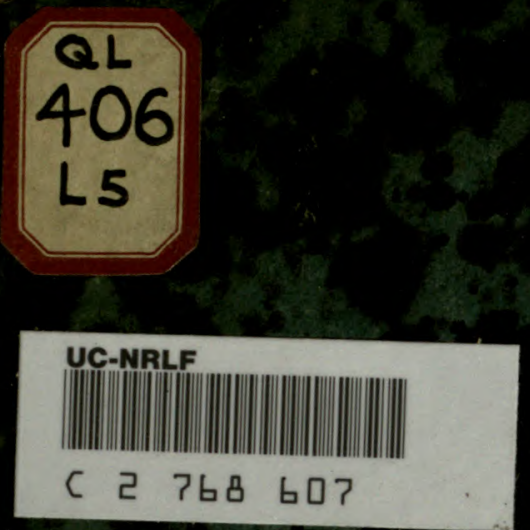

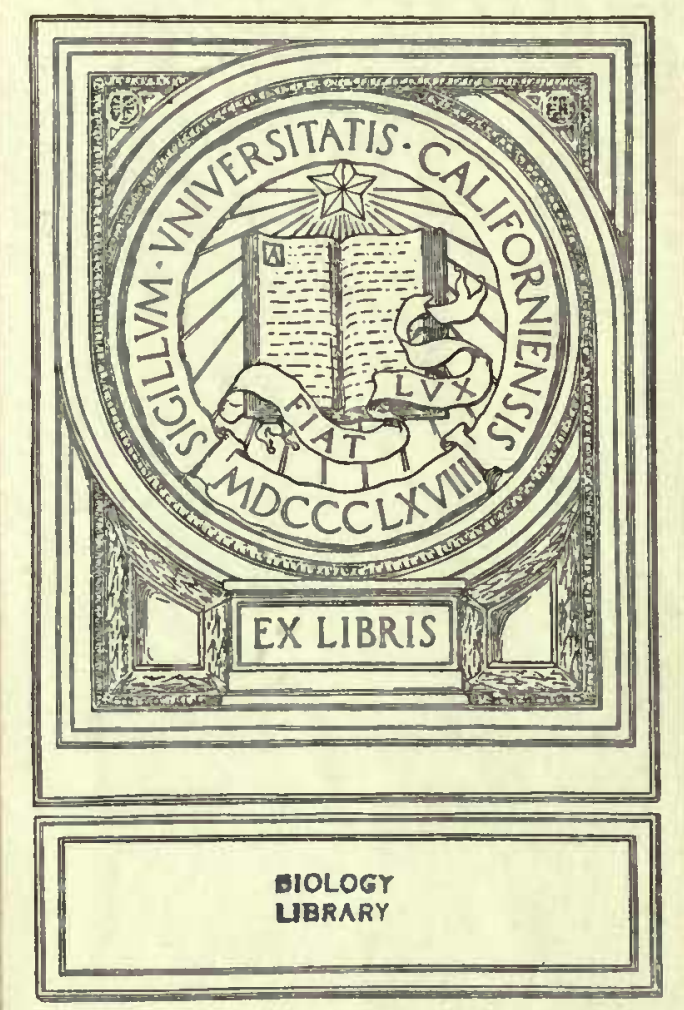


$$
\text { Jo } 2 .
$$



"A hat of shells, marty American, with notations of the places in which They were found and references to works which contain descriptions of them I

This world has not been - identified.

p. $1-52$ missing. 

lit

arctica, lsay.

Beleheii, stinids.

receue, a ad.

ruceata, Stimp.

calata, stinds.

bhuva, Gray.

complanate mode.

craesa, Ainde.

eriefor, Aindes,

cuneata, Sunb.

decens, acad.

delectabilis, a.ad.

ebunnea, sub.

Elenemsis, sour.

excavata, Ninele.

fabula, sowb.

fasligiva, a.ad.

fulgida, a ad,

qubbesa, soub.

hypecborea, Levén.

ircunepicua, a. ad.

inisnata, a. Ad.

Nuculana Link

lata, stindas

cepida, A.ad.

lugubri, a.ad.

lyratw, Ainde. micane, arad. nasula, fowb. ornala, Dóre. pesnula, Mviel. plieiferw, a.ad. polita, font.

puellato, Hinds. recta, siriels. semes ileatw, A. Ad. sevotina, Atinds. siliqua, Reeve. Sowerbyana, D'orb. striata, sowb. sulculata, Gened. tenuis uleata, Cunh. ventricesa, thinde. virew, Dide.

Tamaicensís, dérb.

Adrana.

concinna, $A$. Ad.

decera, A. Ad.

electa, A. ad.

elengata, sowb,

slonina a. Ad.

м̄94103

Patagonica, D'orb. 
arrctica, ypay.

Uldiá.

glavinlis, gray. - s.e. Portlandiá ad il 062

apmica, ad rnve.

laeviata, spengl.

lepidulo, A.ad.

lucida, Leven.

mülleiv, sray

Nucobarica Lam

obesu; stimp.

pygmaea minnat.

retarw, Divids

splendida, Thil

Enello, thids,

Maeletió

Ghilineis, deem. t126f-6.

Gumingii; a. ad. $\frac{\text { Meilo. }}{\text { Ha.ad }} \leftarrow 126 \mathrm{fy}$. 


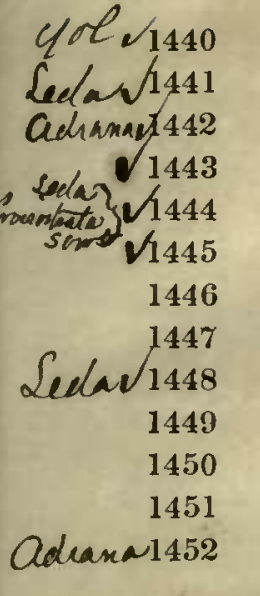

1453

Yre vis4

1455

$\checkmark 1456$

Sedad 1458

1459

$\sqrt{ } 1460$

yed. 1461

$\sqrt{1462}$

1463

Sedad 1465

1466

Ledad 1467

1468
$\sqrt{1469}$

Sedavit70

1471

Cold 1472

1473

$\sqrt{1474}$

1475

Yob $\sqrt{1476}$
Cascoensis,

costellata,

crenifera,

Cumingii,

curvirostra,

delphinodonta,

Deshayesiana,

Eightsii,

emarginata,

“ var.,

fluviatilis,

gigantea,

lanceolata,

laternaria,

lenticula,

limatula,

macilenta,

margaritacea,

Mauritiana,

minuta,

“ var.,

myalis,

navicularis,

nitida,

obliqua,

ovum,

pella,

" var.,

pisum,

Portlandica,

proxima,

recurva,

reticulata,

sapotilla:

tenuis,

tenuis,

Thraciæformis,

tumida,
Mighels,

Sowb., 508 Panama,

Sowb., 507 Xipixapi,

Hinds, Philippines,

Humph., Guinea,

Mighels, Casco Bay,

Duchatel, Anvers,

Couth.,

Lam.,

Sowb.,

508

Sandwich Isles,

Bordeaux,

Greece,

Bengal,

Lam., 504 Xipixapi,

Val., Pacific,

Möll., Greenland,

Say,

Stp.,

Lam.,

Sowb.,

Gmel.,

Couth.,

Couth.,

Sowb.,

Lam.,

Sowb.,

Lam.,

Sowb.,

Hitch.,

Say,

Conrad,

Hinds,

Gould,

Mont.,

Turt.,

Gray,

Storer,

Hinds,

Rhode Island,

Greenland,

506 England,

Singapore,

Massachusetts,

Greenland,

Maine,

British Coast,

505 Valparaiso,

Yorkshire,

505 Mediterranean,

Valparaiso,

Maine,

Liberia,

Philippines,

Cape Cod,

Scotland,

Maine,

Greenland,

Cape Cod,
Massachusetts Bay, Bost. Jour., 2, pl. 3, f. 7 .

Massachusetts Bay, Gould's Invert., p. 103, f. 63.

St. of Malacca,

Gould's Invert., p. 98, f. 62.

Index Möll., p. 17.

Sowb. C. III., f. 17, spec. 22.

Index Möll., p. 17.

Bost. Jour., 2, pl. 4, f. 4.

Sowb. C. 1ll., f. 20, spec. 29.

Sowb. C. Ill., f. 21, spec. 20.

Sowb. Gen., f. 4

Sowb. C. Ill., f. 23, spec. 33.

Zool. Proc., 1843, p. 100.

Gould's Invert., p. 100, f. 61.

Ind. Möll. Groenl., p. 17.
Bost. Jour., 4, pl. 4, f. 6.

Sowb. C. Syst., pl. 85, f. 8.

Sowb. C. Illust., f. 3.

Zool. Sulph., p. 62, pl. 18, f. 1 .

Sowb. C. Illust., 7, f. $5 . \quad$ Area rostrata, Mont.

Bost. Jour., 4, p. 40, pl. 4, f. 5.

Jay's Cat., 1839, pl. 1, f. 12. Nucula laternaria ? Val.

Desh. Tr. El., pl. 34, f. 8-10. fossil.

Poli, pl. 25, f. 4, 5.

Sowb. Gen., f. 3.

Sowb. Gen., f. 1.

Voy. Venus, pl. 33, f. 5. is Nucula Eightsii ? Couth.

Index Möll., p. 17.

Don. B. Shells, 2, pl. $63 . \quad$ Donax argentea, Gmel.

Bost. Jour., 2, pl. 3, f. $8 . \quad$ Nucula tenuisulcata, Couth.

Sowb. Min. C., pl. 476, f. 1. fossil.

Chemn. 7, pl. 55, f. $546 . \quad$ fossil.

Bost. Jour. N. H., 1, p. 327. fossil.

Jour. Ac. N. S., 6, pl. 11, f. 21. Nucula curvirostra? Humph.

Test. Brit. Supp., pl. 29, f. 1. Arca tenuis, Mont.

Gould's Invert., p. 105, f. 64. Nucula lucida, Blanding.

Bost. Jour., vol. 2, p. 122. = S.G. Brllandice Qd in' 6652

Zool. Sulph., p. 63, pl. 18, f. 6. 
Chass. TROPIOPODA.

\author{
ORDER. B I M U S C U L OSA.
}

FAMILX TRIGONACEA.

GENUS TR1GONIA. Bruguière.

1478 alæformis, Sowb., 515 France,

a) Maugund479 pectinata,

\begin{abstract}
Lam., 514 N. Holland,
\end{abstract}
Sowb. Gen., f. 1, 2.

Voy. Astrol., pl. 78, f. 1-4. fossil, Trigonia crenulata.

Trigonia margaritacca, Lam.

\title{
CLAs S TROPIOPODA.
}

Order. BIM U S C ULOSA.

$$
\text { FAMIIX NAIADES. }
$$

GENUS UN10. Bruguière.

1481

1482

1483

1484

1485

1486

1487

1488

1489

1490

1491

1492

1493

1494

1495 abruptus,

acutissimus,

Esopus,

Alabamensis,

alatus,

" var.,

" "

"

altilis,

ambiguus,

$$
\text { " va }
$$

(4) " "

$"$

"

amnicus,

angustatus,

Anodontina,

Anodontoides,

apiculatus,
Say,

Lea,

Green,

Conrad,

Say,

Conrad,

Lea, "

$2+3$
555 Wabash River, Alabama River, Ohio River, Alabama River,

539 Fox River, Detroit River, N. West Territory,

Ohio, Alabama River,

523 S. America, Amazon River, Corrientes, Argent. Republic, Bolivia, Rio de la Plata, Carniola, Congaree River,

Lea, Lam., Lea, Say,

546 Bengal, 547 Ohio River, N. Orleans,
Amer. Conch., pl. 17. Tr.Am. P.S.,4,p.89,pl.10,f.18.

is Unio crassus, Say. C. of Mac. Lyc., 1827, 1, pl. 3. in Unio cyphius, Raf. N. F. W. Shells, p. 67. Nich. Ency. Am., pl. 4, f. 2. is Unio inflatus, Lea. Conr. Monog., p. 56, pl. 31. Unio megaptera, Raf. Sowb. C. Syst., pl. 89, f. 8. Swain's Exot. C., pl. $7 . \quad$ Unio alatus, Swains. N. F. W. Shells, p. 43, pl. 2, f. 1. Sowb. C. Syst., pl. 87, f. 2 . Castalia ambigua, Lam. Blain. Mal., pl. 67, f. 4. Unio ambigua, Blain. Cuv. Regn. An., pl. 90 bis? f. 2. Mya ambigua, Wood. Spix. Test., p. 32, pl. 25, f. 3, 4. Tetraplodon pectinatum, Spix. , Voy. Amer. M., pl. 73. Castalia quadrilatera, D'Orb.

Voy. Aıner. M., pl. 72, f. 4-10. Castalia inflata, D'Orb. Rossm., pl. 15, f. 212. Unio batavus, Lam. Ts. $\Lambda$ m. P. S., v. 4, pl. 17, f. 43.

Enc. Meth., pl. 247, f. 1. is Unio marginalis, Lam. Ts. Am. P. S., v. 4, pl. 8, f. 11. is Unio teres, Raf.

Amer. Conch., pl. 52. 
Unjoincias

Lumarckir, lray nobilis, A. Ad.

Stangei, A.ad.

uniophora, emay.

ornata, Dóre.

Verticordiá 



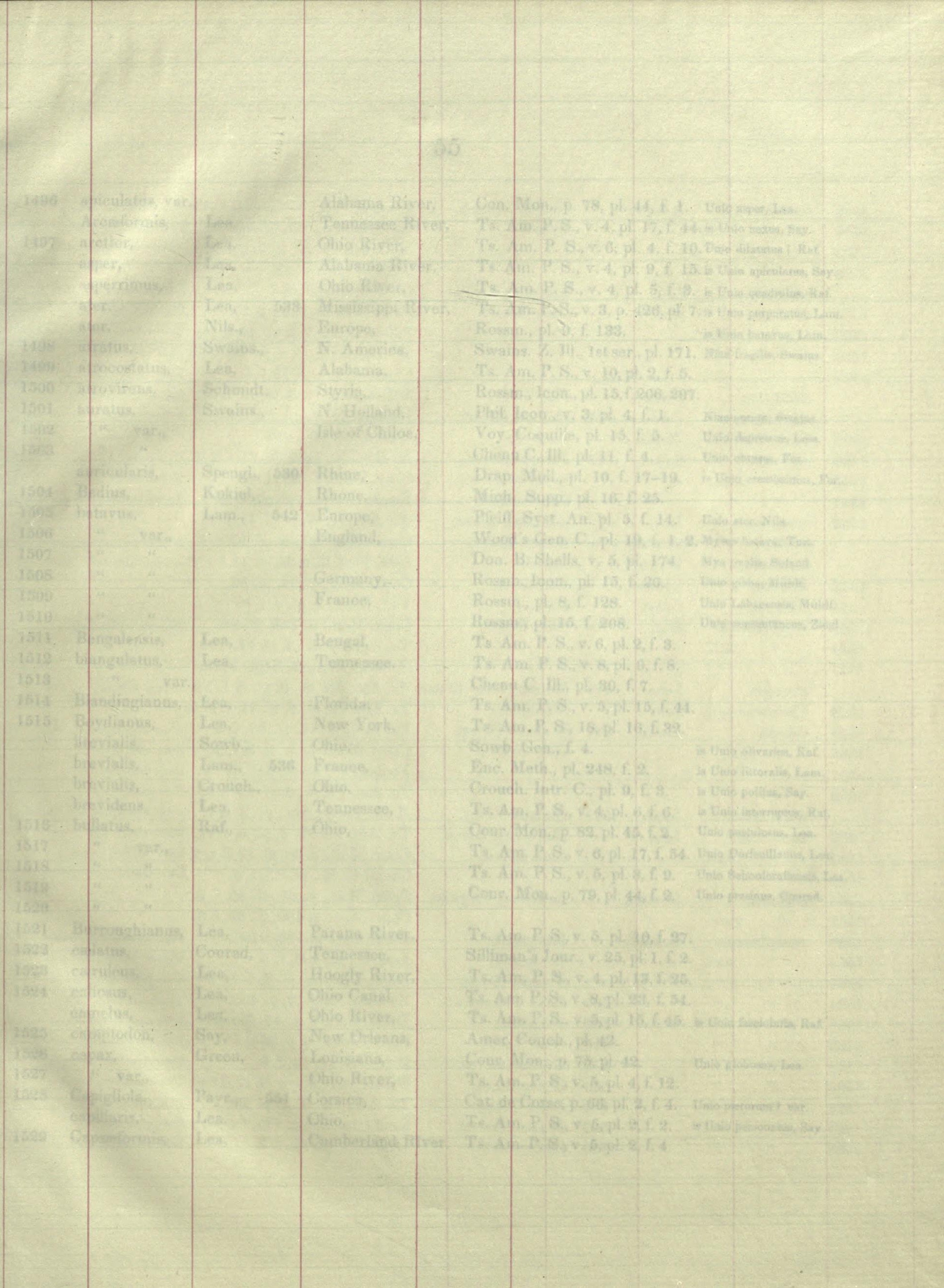



1496 apiculatus, var.,

1497

1498

1499

1500

1501

1502

1503

1504

1505

1506

1507

1508

1509

1510

1511

1512

1513

1514

1515

1516

1517

1518

1519

1520

1521

1522

1523

1524

1525

1526

1527

1528

1529

" var.,

auricularis,

Badius,

batavus,

" var.,

" "

" "

" "

" "

brevialis,

brevialis,

brevialis,

brevidens,

bullatus,

" var.

" "

" "

" "

cælatus,

cæruleus,

callosus,

camelus,

camptodon,

capax,

" var.,

Capigliola,

capillaris,
Arcæformis, Lea,

arctior, Lea,

asper, Lea,

asperrimus, Lea,

ater, Lea,

ater, Nils.,

atratus, Swains.,

atrocostatus, Lea,

atrovirens, Schmidt,

auratus, Swains.,

Bengalensis, Lea,

biangulatus, Lea, var.,

Blandingianus, Lea,

Boydianus, Lea,

Burroughianus, Lea,

Capsæformis, Lea,

Lea,

Raf.,
Alabama River, Con. Mon., p. 78, pl. 44, f. 1. Unio asper, Lea.

Tennessee River, Ts. Ain. P.S., v. 4, pl. 17, f. 44. is Unio nexus, Say.

Ohio River,

Alabama River,

Ohio River,

'T's. Am. P. S., v. 6, pl. 4, f. 10. Unio dilatatus? Raf.

Ts. Am. P. S., v. 4, pl. 9, f. 15. is Unio apiculatus, Say.

Ts. Am. P. S., v. 4, pl. 5, f. 3. is Unio quadrulus, Raf.

533 Mississippi River,

Ts. Am. P. S., v. 3, p. 426, pl. 7. is Unio purpuratus, Lam.

Europe,

Rossm., pl. 9, f. 133.

is Unio batavus, Lam.

N. America,

Alabama,

Styria,

N. Holland,

Isle of Chiloe,

Spengl., 530 Rhine,

Kokiel, Rhone,

Lam., 542 Europe,

England,

Germany,

France,

Swains. Z. Ill., 1st ser., pl. 171. Niaa fragilis, Swains.

Ts. Am. P. S., v. 10, pl. 2, f. 5.

Rossm., Icon., pl. 15, f.206, 207.

Phil. Icon., v. 3, pl. 4, f. 1. Niaa aurata, Swains.

Voy. Coquille, pl. 15, f. 5. Unio depressus, Less.

Chenu C. Ill., pl. 11, f. $4 . \quad$ Unio obtusus, Fer.

Drap. Moll., pl. 10, f. 17-19. is Unio erassissimus, Fer.

Mich. Supp., pl. 16, f. 25.

Pfeiff. Syst. An. pl. 5, f. 14 . Unio ater, Nils.

Wood's Gen. C., pl. 19, f. 1, 2. Mysea batava, Turt.

Don. B. Shells, v. 5, pl. 174. Mya ovalis, Soland.

Rossin. lcon., pl. 15, f. 20. Unio gibba, Müblf.

Rossm., pl. 8, f. 128.

Rossm., pl. 15, f. 208.

Unio Labaeensis, Mühlt:

Unio consentancus, Ziegl.

Bengal,

Tennessee,

Florida,

New York,

Ohio,

Lam., 536 France,

Crouch., Olio,

Tennessee,

Ohio,

Ts. Am. P. S., v. 6, pl. 2, f. 3.

Ts. Am. P. S., v. 8, pl. 9, f. 8.

Chenu C. Ill., pl. 30, f. 7 .

Ts. Am. P. S., v. 5, pl. 15, f. 44

Ts. Am. P. S., 18, pl. 16, f. 32.

Sowb. Gen., f. 4.

is Unio olivarius, Raf.

Enc. Meth., pl. 248, f. $2 . \quad$ is Unio lilloralis, Lam.

Crouch. Intr. C., pl. 9, f. 3. is Unio politus, Say.

Ts. Am. P.S., v. 4, pl. 6, t. 6. is Unio interruptus, Rat.

Conr. Mon., p. 82, pl. 45, f. 2 . Unio pustulosus, Lea.

Ts. Am. P. S., v. 6, pl. 17, f. 54. Unio Dorfcuillanus, Lea.

Ts. Am. P. S., v. 5, pl. 3, f. 9 . Unio Schoolcraliensis, Lea.

Conr. Mon., p. 79, pl.44, f. 2 . Unio prasinus, Conrad.

Ts. Am. P. S., v. 5, pl. 10, f. 27.

Silliman's Jour., v. 25, pl. 1, f. 2.

T's. Am. P. S., v. 4, pl. 13, f. 25.

Ts. Am. P. S., v. 8, pl. 23, f. 54.

Ts. Am. P. S., v. 5, pl. 15, f. 45. is Unio fasciolarios Ra?.

Amer. Conch., pl. 42.

Conr. Mon., p. 75, pl. 42.

Unio globosus, Lea.

Ts. Am. P. S., v. 5, pl. 4, ł. 12

Cat. de Corse, p. 66, pl. 2, f. 4. Unio piclorum? var.

Ts. Am. P. S., v. 5 ; pl. 2 , f. 2 . is Unio personatus, Say.

Ohio,

Cumberland River, Ts. Am. P. S., v. 5, pl. 2, f. 4. 


\begin{tabular}{|c|c|c|c|c|c|c|}
\hline 1530 & carbonarius, & Lea, & & Medellin River, & Am. P. S., v. 6, pl. 11, f. 32. & Unio pliciferus, Lea. \\
\hline 531 & " var., & & & Mexic & Mon., p. 89 , pl. 41 , f. 1. & Unio plexus, Conrad. \\
\hline 1532 & " & & & & Phil. Icon., p. 19, pl. 1, f. 1, 2,3 & Unio scmigranosa, Busch. \\
\hline 1533 & “ & & & & Chenu C. Ill., pl. 23, f. I. & \\
\hline & cardiacea, & Say, & & River, & Guer. Icon., pl. 28, f. 7. & is Unio Mytiloides, Raf. \\
\hline 1534 & cardium, & & & er, & Transl. Raf., & Unio occidens, Lea. \\
\hline 1535 & "var., & & & & Sillim. Jour., v. 6, pl. 13, f. 14. & Unio ventricosus, Barnes, Say \\
\hline 1536 & “ & & & & Ts. Am. P. S., v. 4, pl. 18, f. 46. & Unio subovatus, Lea. \\
\hline 1537 & “ & & & & Chenu C. Ill., pl. 13, f. 5. & Unio ventricosus, Say. \\
\hline & carinatus, & arnes, & & Fox River, & Sillim. Jour., v. 6 & is Unio fasciatus, Raf. \\
\hline & carinifera, & Lam., & 536 & Hudson River, & N. Hist. N. Y., & is Unio complanatus, Soland \\
\hline 1538 & Carinthiacus, & Ziegl., & & Carniola, & Rossm., pl. 15, f. 209. & Unio batavus, Lam. ex Lea. \\
\hline 1539 & cariosus, & Say, & 545 & Passaic River, & N. Hist. N. York, pl. 21, f. 243. & - Unio ovata, Valenc. \\
\hline 1540 & " var., & & & Hudson River, & Conr. Mon., p. 40, p & Unio luteolus, Lam. \\
\hline 1541 & “ & & & Connecticut, & Reeve C. Syst., pl. 89, f. 10. & Unio luteolus, Conrad. \\
\hline 1542 & castaneus, & Lea, & & Alabama River, & Ts. Am. Ph. S., 4, pl. 11, f. 21. & \\
\hline & catillus, & Conrad, & & ta River, & Conr. Mon., p. 30, pl. 13, f. 2 . & is Unio coccineus, Lea. \\
\hline & caudatus, & Wagn., & & & Spi & is \\
\hline 1543 & cerinus, & Conrad, & & siana, & Conr. Mon., p & \\
\hline 1544 & Chilensis, & Gray, & & $\mathrm{Cl}$ & Phil. Icon., & \\
\hline 1545 & cicatricosus, & Say, & & Ohio River, & Conr. Mon., p. 115, pl. 64. & Unio varicosus, Lea. \\
\hline 1546 & " var., & & & & Ts. Am. Ph. S., 4, pl. 11, f. 20. & \\
\hline 1547 & “ & & & & N. Har. Diss., 2, $\mathrm{S}$ & \\
\hline & circulus, & Lea, & 549 & er, & Ts. A & is Unio subrotundus, Ral: \\
\hline 1548 & Claibornensis, & Lea, & & & 115 . & \\
\hline 1549 & clavus, & Lam., & 537 & Wabash River, & Reeve C. Syst., pl. 88, f. 3. & Unio Modiolilormis, Say. \\
\hline 1550 & " var., & & & Ohio River, & Conr. Mon., p. 5, pl. 3, f. 1. & Unio scalenius, Raf. \\
\hline 1551 & " & & & & Poulson's Tre & Unio cuneatus, Raf. \\
\hline 1552 & “ & & & & Ts. Am. Ph. S., 3, pl. 12, f. 20. & Unio patulus, Lea. \\
\hline & coarctatus, & Lam., & 535 & Hudson River, & Ency. Am., pl, 3, f. 1 . & is Unio complanatus, Soland. \\
\hline 1553 & coccineus, & Lea, & & Mahoning River, & Ph. S., 6, pl. 5, f. 12. & Unio rubra? Raf. \\
\hline 1554 & var., & & & Ohio River, & Conr. Mon., p. 30, pl. 13, f. 1, 2. & Unio catillus, Conrad. \\
\hline 1555 & “ & & a & & Chenu C. IIl., $p$ & Unio Gouldianus? Ward. \\
\hline 1556 & collinus, & Conrad, & & N. River, Virginia, & Conr. Mon., p. 65, pl. 36, f. 2. & \\
\hline 1557 & “ var., & & & & Conr. Mon., p. 109, & \\
\hline 1558 & complanatus, & Soland, & & Maryland, & Portl. Cat., p. 100, 2190. & Mya complanata, Soland. \\
\hline 1559 & var., & & & N. Jersey, & List. C., pl. 150 , f. 5. & Unio violaceus, Spengler. \\
\hline 1560 & “ & & 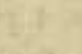 & Yadkin River, & Nich. Encyc & Unio purpureus, Say. \\
\hline 1561 & “ & & 534 & Lake Champlain, & Schroet. Einl., 2, p. 458. & Unio rarisulcata, Lam. \\
\hline 1562 & “ & & 535 & Virginia, & Dillw, Cat., 1, p. 51, No. 27. & Unio coarctata, Lam. \\
\hline 1563 & “ & & 535 & New York, & Sillim. Jour., 6, p. 264, No. 16. & Unio purpurascens, Lam. \\
\hline 1564 & “ & & 536 & Hudson River, & Delessert, pl. 12, f. 8 . & Unio rhombula, Lam. \\
\hline 1565 & “ & & 536 & Connecticut, & Nat. Hist. N.York, pl. 22, f. 246 & Unio cariniffera, Lam. \\
\hline 1566 & " & & 536 & Lake George, & Ts. Am. Ph. S., 6, p. 264 & Unio Georgina, Lam. \\
\hline 1567 & “ & & 537 & Massachusetts, & Gould Invert., p. 117, f. 68-70. & Unio glabrata, Lam. \\
\hline 1568 & " & & 540 & Connecticut, & Delessert, pl. 12, f. 3. & Unio suleidens, Lạm. \\
\hline
\end{tabular}








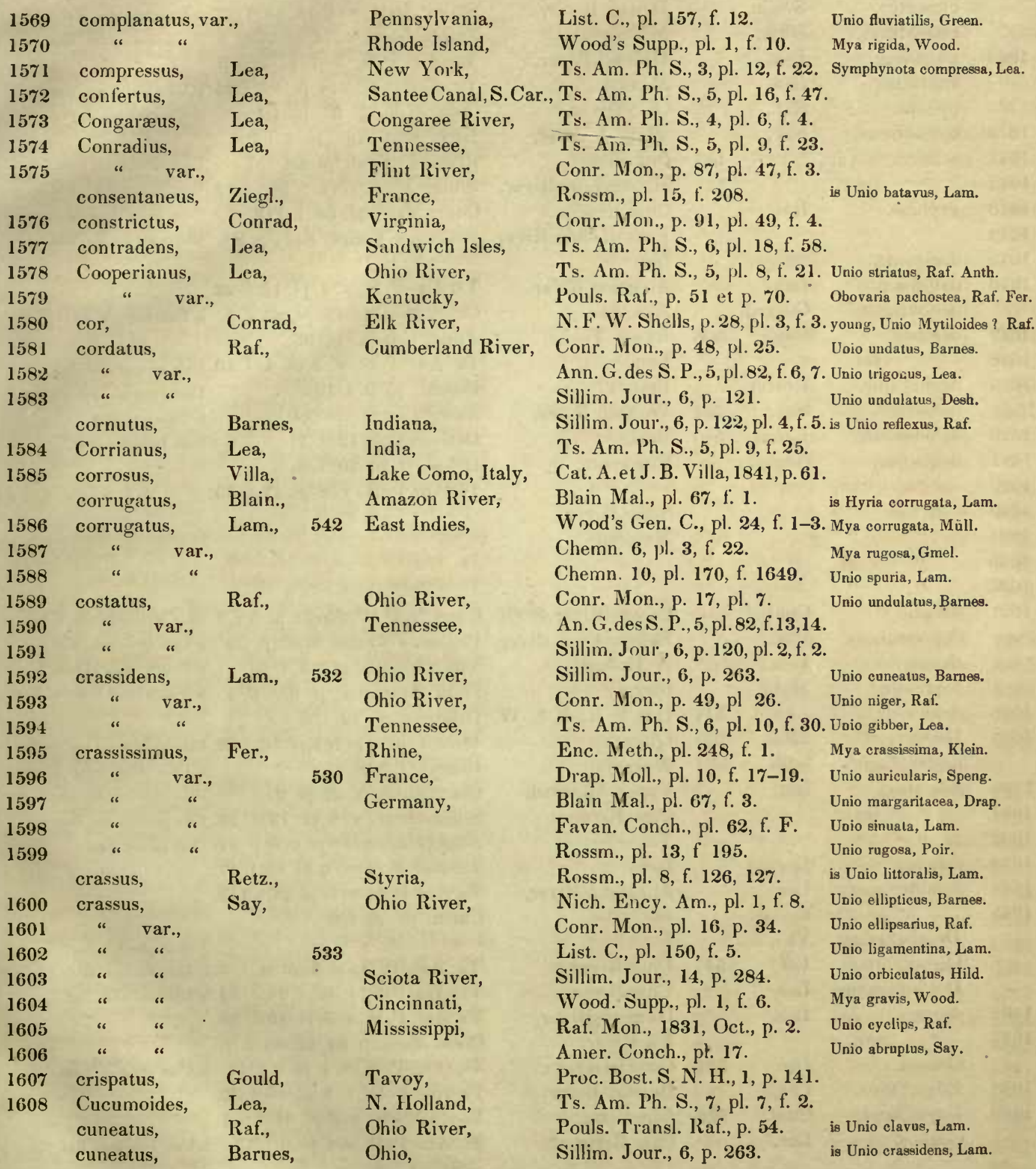




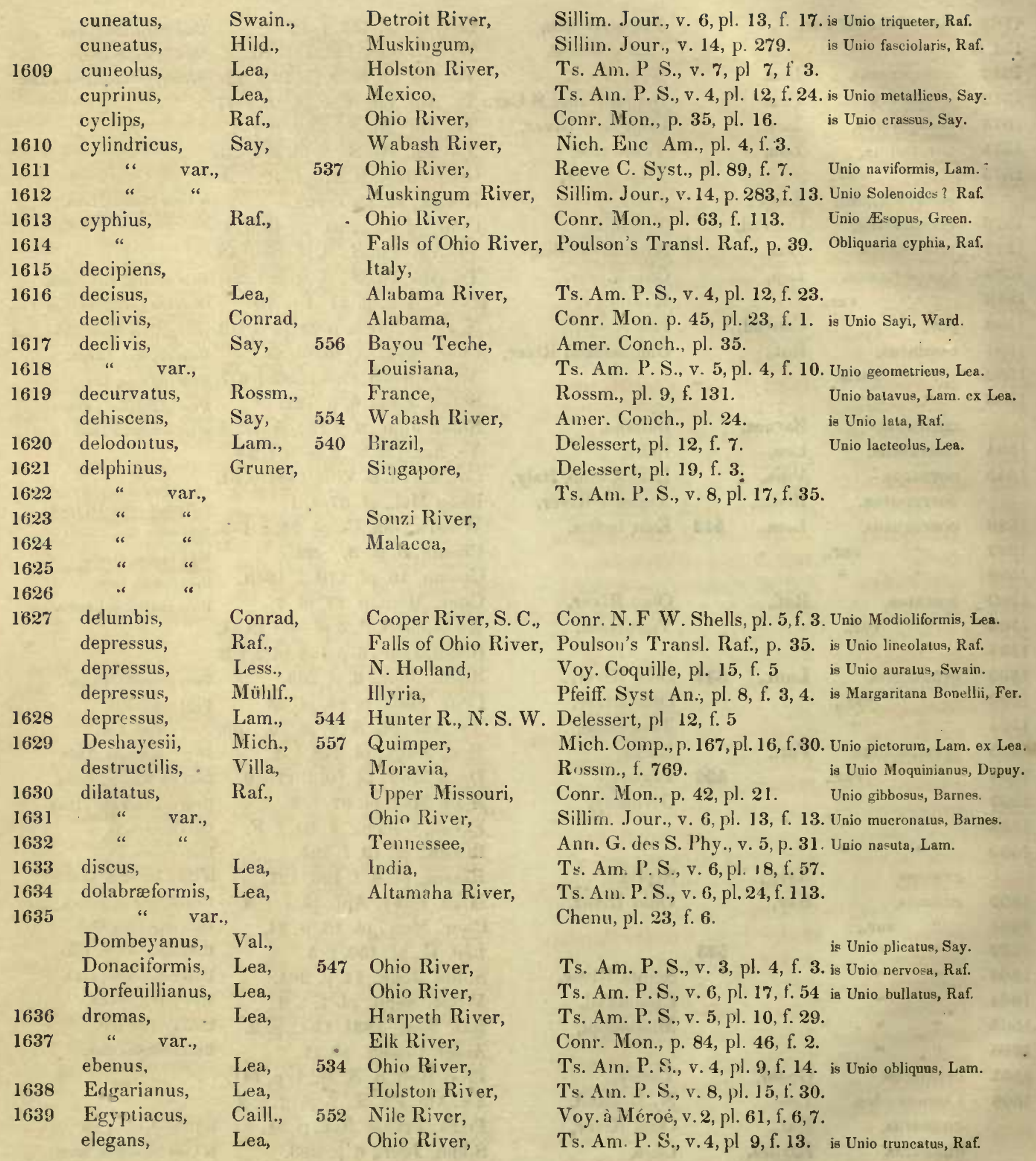






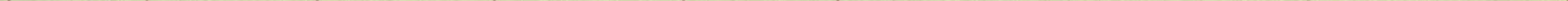



164

1641

$164 \%$

1643

1644

1645

1646

1647

1648

1649

1650 .

1651

1652

1653

1651

1655

1656

1657

1658

1659

1660

1661

1662

1663

1664

1665

1666

1667

1668

1669
Raf.,

ellipsarius,

ellipsis,

ellipticus,

ellipticus,

elongatulus,

elongatus,

emarginatus,

errosus,

excultus,

exolescens,

fabalis,

fasciatus,

" var., " “"

fasciolaris, Raf., " var., " "

fasciolus, " var.,

Fisherianus

flavus, " var.,

flexuosus, " var., fluviatilis,

foliacea,

foliatus,

folliculatus, Lea,

formosus,

fragilis,

fragilis, " var.,

“"

fragosus,

fulvus, var.,

fusculus, geometricus,

Georgina,

gibba, gibber, gibbosus, “. var. gibbosus,

Lea,

Lam.,

Lea,

Retz.,

Gould,

Lea,

Raf.,

Raf.,

Lea,

Raf.,

Raf.,

Hild.,

Raf.,

Lea,

Lea,

Lea,

Raf.,
Wagn.,

Barnes,

Miillf.,

Conrad,

Green,

Gould,

Swains., 559

Conrad,

Ziegl.,

Lam.,

Mühlf.,

\section{9}

Ohio River,

Olio River,

Wabash River,

Chester River,

Kentucky,

Ohio River,

Ohio River,

New York,

Tavoy,

Ohio River,

Savannah River,

Ohio River,

Ohio River,

Detroit River,

Fox River,

Ohio River,

Sciota River,

South Carolina,

Styria,

Louisiana,

536 Lake George,

542

Germany,

Tennessee,

Ohio River,

Sciota River,

Wabash River,

Barnes,
Pouls. Transl. Raf., p. 35. is Unio crassus, Say.

Ts. Am. P. S., v. 3, pl. 4, f. 4. is Unio olivarius, Raf.

Spix Test. B., pl. 26, f. 1, 2. is Unio multistriatus, Lea.

Sillim. Juur., v. 6, p. 259, f. 19. is Unio crassus, Say.

Rossnt. Icon., pl. 9, f. 132. is Unio pietorum, Lam. ex Lea.

Pfciff. L. \& S. Möll., pl. 5, f 11. is Margaritana margaritifera.

'îs. Am. P. S., v. 5, pl. 9; f. 22.

Unio littoralis? Pfeiff.

Conr. Mon., p. 99, pl. 55, f. 1.

Proc. Bost. S. N. H , v. 1, p. 141.

Ts. Ain. P. S., v. 4, pl. 10, f. 16. is Unio lapillus, Say.

Conr. Mon., p. 3, pl. $1 . \quad$ Unio earinalus, Barnes.

Sillim. Jour., v. 6, pl. 11, f. 10.

Ann. Gen. des S. P., v. 5, p. 28.

A mer. Conch., pl. 22. Unio phaseolus, Hild.

Ts. Am. P. S., v. 5, pl. 9, f. 13. Unio planulatus, Lea.

Ts. Am. P. S., v. 5, pl. 15, f. 45. Unio eamelus, Lea.

Sillim. Jour., v. 14, p. 279, 283. Unio cuneatus, Hild.

Conr. Mon., p. 26, pl. 11, t. 2. Unio multiradiatus, Lea.

Ann. Gen.des S. P., v. 5, p. 33. Unio ligamentina, Desh.

Ts. Am. P. S., v. 6, pl. 4, f. 8.

Conr. Mon., p. 74, pl. 41, f. 2. Unio rubiginosus, Lea.

'Ts. Am. P. S., v. 3, pl. 8, f. 10.

Conr. Mon., p. 8, pl. 4, f. 2 . Unio foliatus, Hild.

Sillim. Jour., 14, p. 248, f. 16.

List. C., pl. 157, f. 12.

is Unio complanatus, Solan.

Proc. Bost. S. N. II., 1, p. 141.

Conr. Mon., p. 8, pl. 4, f. 2. is Unio flexuosus, Raf.

Ts. Am. Ph. S., 6, pl. 11, f. 33.

Ts. Am. Ph. S., 4, pl. 16, f. 41. is Unio Iriqueter, Raf.

Zool. Ill., 1st Ser., 3, pl. 171. is Unio fragilis, Raf.

Conr. Mon., p. 55, pl. $30 . \quad$ Unio gracilis, Barnes.

Zool. 11l., 3, pl. 171.

Unio fragilis, Swains.

'Ts. Am. Ph. S.. 3, p. $432 . \quad$ Symphynota gracilis, Lea.

Conr. Mon, p. 12, pl. 6, f. 2.

Ts. Am. Ph. S., 5, pl. 13, f. 39. Unio icterinus, Conrad.

Rossm. Icon., pl. 15, f. 211.

'Ts. Am. Ph. S., 5, pl. 4, f. 10. is Unio declivis, Say.

Ts. Am. Ph. S., 3, p. 416 . is Unio complanatus, Soland.

Rossm., f 20.

is Unio batavus, Lam.

Ts. Atn. Ph. S., 6, pl. 10, f. 30. is Unio crassidens, Lam.

Pouls. 'Transl. Raf., p. 56.

Ts. Am. Ph. S., 4, pl. 17, f. 42. Unio torulosus, Raf.

Conr. Mon., p. 50, pl. 27, f. 1 Unio perplexus, Lea.

Conr. Mon., p. 42, pl. $21 . \quad$ is Unio dilatatus, Raf. 


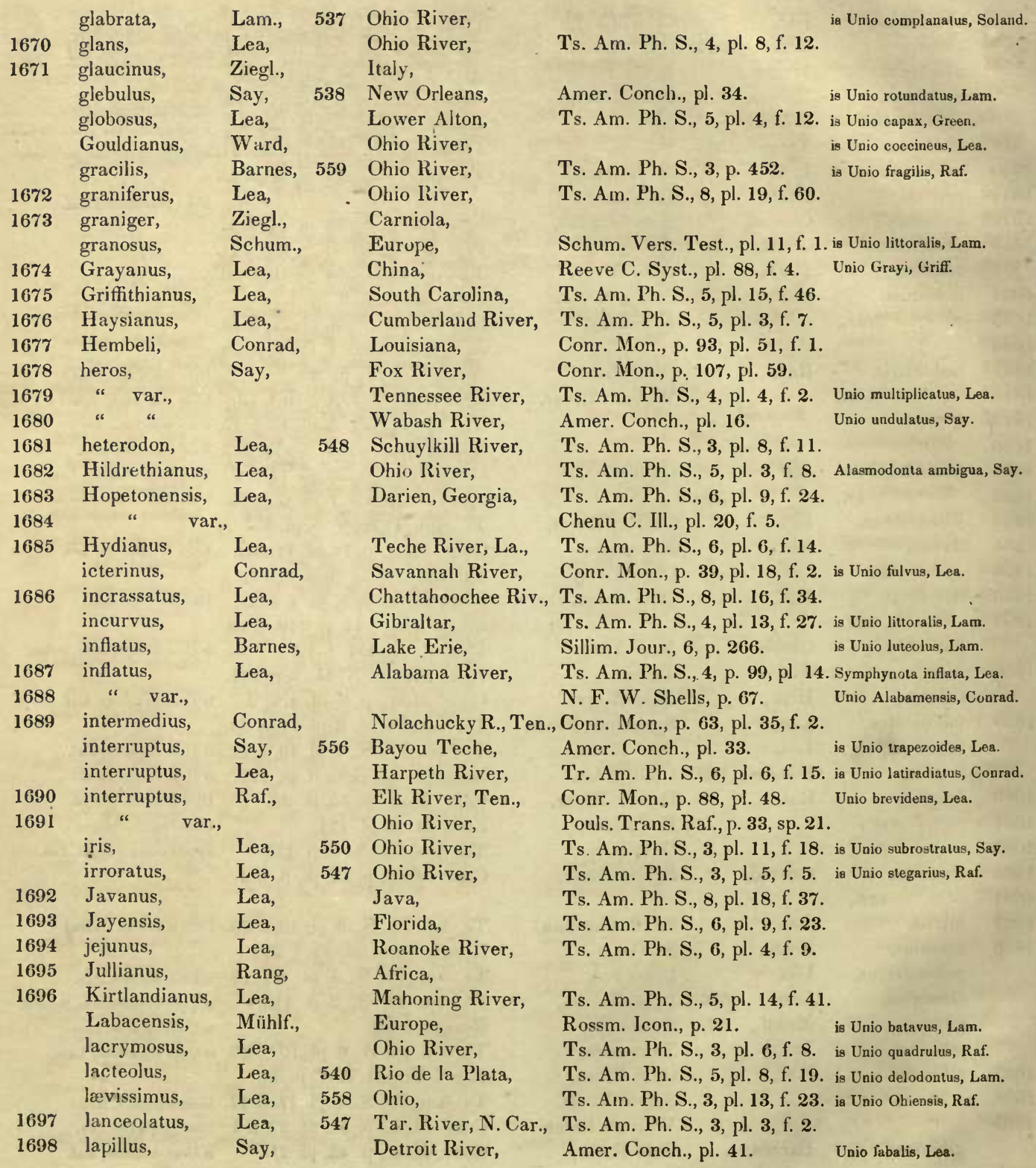








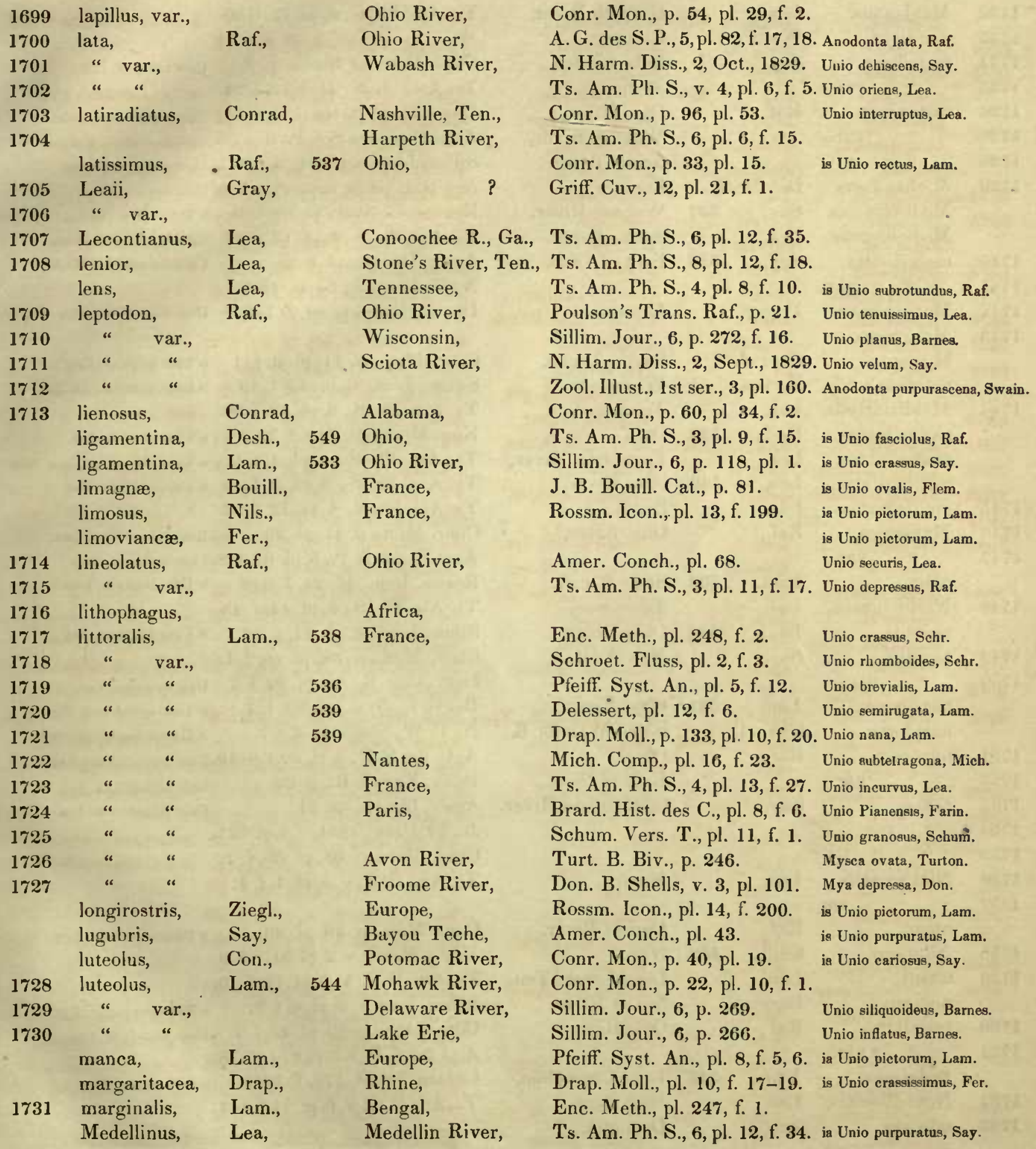




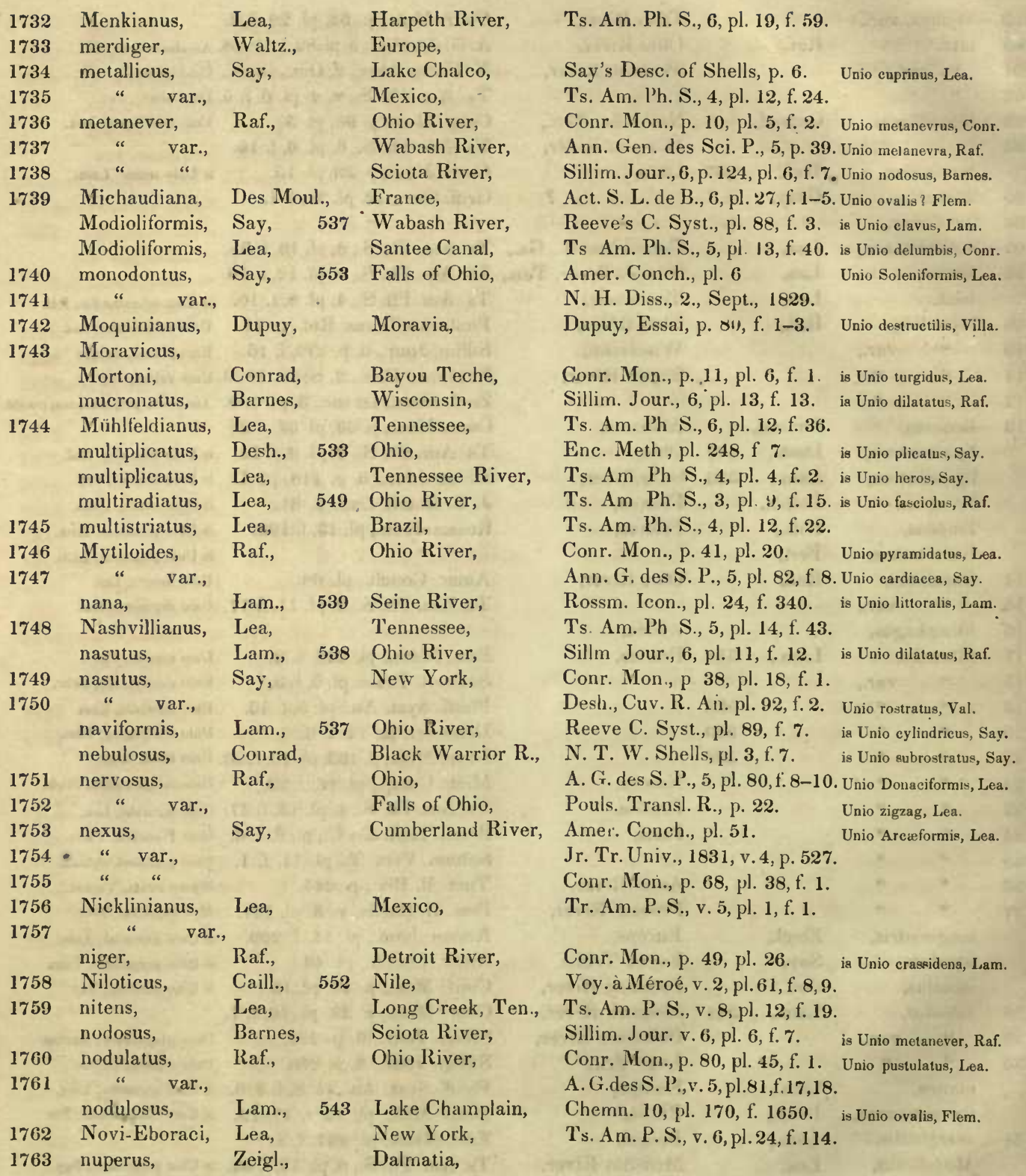








\begin{tabular}{|c|c|c|c|c|c|}
\hline 64 & obesus, & ea, & York River, Vir., & Ts. Am. P. S., v. 4, pl. 13, f. 26. & \\
\hline 65 & obliquatus, & Raf., & Ohio, & Ann. Gen. des S. P., v. 5, p. 43. & \\
\hline 766 & var., & 10 & Kentucky, & N.H.Diss, v.2, p.308, Oct.1829. & Unio ridibundus, Say. \\
\hline 67 & “ & 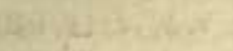 & 11 & Ts. Am. P. S., v. 3, pl. 8, f. 12. & Unio sulcatus, Lea. \\
\hline & obliquus, & Lam., $\quad \mathbf{5 3 4}$ & Ohio River, & Conr. Mon., p. 77, pl. 43, f. 2. & Unio obovalis, Raf. \\
\hline & var., & & & T's. Am. P. S., v. 3, pl. 9, f 14. & Unio ebenus, Lea. \\
\hline & obovalis, & Raf., & Ohio River, & . 45. & is Unio obliquus, Lam. \\
\hline & obtusus, & Fer., & N. Holland, & 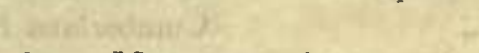 & is Unio auralus, Swains. \\
\hline & & Desh., $\quad 538$ & hannah R., & Conr. Mon., p. 4, pl. 2. & is Unio ovatus, Say. \\
\hline & & Lea, & Ohi & S., v. 3, pl. 10, f. 16 . & is Unio cardium, Raf. \\
\hline 70 & Ohiensis, & Raf., & Ohio & Ann. Gen. des S. P., v. 5, & Anodonta Ohiensis, Rat \\
\hline 71 & 14. & $36=51 x$ & 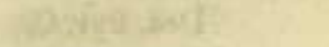 & Ts. Am. P. S., v. 3, pl. 13 & Unio lævissimus, Lea. \\
\hline 72 & ochraceus, & Say, & Passaic River, & Nich. Ene Amer., & theting \\
\hline 73 & " var., & & ersey, & Conr. Mon., p. 37, pl. 17, f. 2. & Symphynota ochracea \\
\hline 74 & 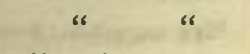 & at & ill River, & Lis & Mylilus fluviatilis, Gme \\
\hline 75 & olivarius, & Raf., & Ohi & Ts. Am. P.S., v. 3, pl. 4, f. 4. & * \\
\hline 76 & var., & & ky, & Ann. Gen. des S. P., v. 5, p. 48. & - \\
\hline 77 & “ & & ssee, & Pouls. Transl. Raf., p. 55. & Unio ellipsis, Lea. \\
\hline 78 & olivarius, & Lea, & River, 1 & S., v. 4, pl. 16, f. 38. & \\
\hline & orbiculatus, & Hild., $\quad 555$ & Mu & Sil & is Unio crassus, Say.. \\
\hline & oriens, & Lea, $\quad 554$ & & 6, f. 5. & is Unio lata, Raf. \\
\hline 79 & ovalis, & Flem., & Eng & List. C., pl. 147, f. 2. & Mytilus angustior, List. \\
\hline 80 & var., & 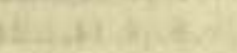 & 10 & Mont. Test & Mya ovalis, Mont. \\
\hline 81 & & & & ls, v 4 , pl. 122 & Mya ovata, Donov. \\
\hline 82 & “ & & & olls, v. 3, & Mya depressa, Donov. \\
\hline 83 & “ & & Av & B Biv., pl. 16, f. 2 & Myeca solida, Turt. \\
\hline 84 & “ & 543 & Lake Champlain, & Chemn. 10, pl. 170, f. 1650 & Unio nodulo:a, Lam. \\
\hline 85 & & 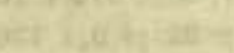 & & 1., pl. 3, f. 70, $a, b$ & Unio lumida, Retz. \\
\hline 86 & “ & 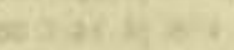 & 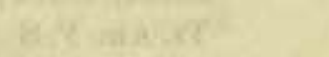 & 202-204. & Unio ovata, Bouil. \\
\hline 87 & & $14^{2} x^{2}$ & $4=$ & J. B. Bouillet Cat., p. 81 . & Unio rostrata, Stud. \\
\hline 888 & & 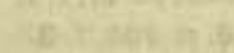 & $y^{3}$ & Mich. Comp., pl. 16, f. 25 & Unio limagnæ, Bouil. \\
\hline 89 & & cat int & 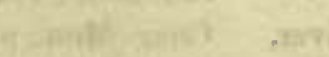 & Wood's Gen. C., pl. 19, f. 5. & . 784 \\
\hline 90 & “ & & & st., pl 87, f. 1. & - \\
\hline & ovata, & Valenc., & $\mathrm{Ne}$ & pl. 89, f. 10. & is Unio cariosus, Say. \\
\hline & ovata, & Bouillet, & Fr & 84, No. 98. & is Unio ovalis, Flem. \\
\hline 791 & ovatus, & 38 & er, & Conr. Mon., p. 4, pl. 2. & Unio ventricosus, Desh. \\
\hline & var., & & (c) & $i^{2}$ & Unio subovatus, Desh. \\
\hline 93 & “ & & & 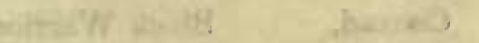 & Unio occidens, Desh \\
\hline 794 & oviformis, & Conrad, & Ten & N. F. W. Shells, p.46, pl. 3, f. 6. & xispevity \\
\hline 795 & paludicolor, & Gould, & les, Flo., & 53. & $*$ \\
\hline & papyrac & Gould, & $\mathrm{Mi}$ & Proc $\mathrm{Bo}$ & $x=$ \\
\hline 797 & paralle] & , Lea, & Parana River, & Ts. Am. P. S., v. 5, pl. 8, f. 20. & 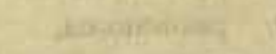 \\
\hline 1798 & Paranensis, & Lea, & Parana River, & Ts. Am. P. S., v. 5, pl. 14, f. 42. & Unio Solisiana, D'Orb. \\
\hline 1799 & parvus, & & & & an nowothis \\
\hline & & & & & A \\
\hline & Patagonicus, & & $\cos , \mathrm{P}$ & $1-4$. & - $-2 a y$ \\
\hline
\end{tabular}




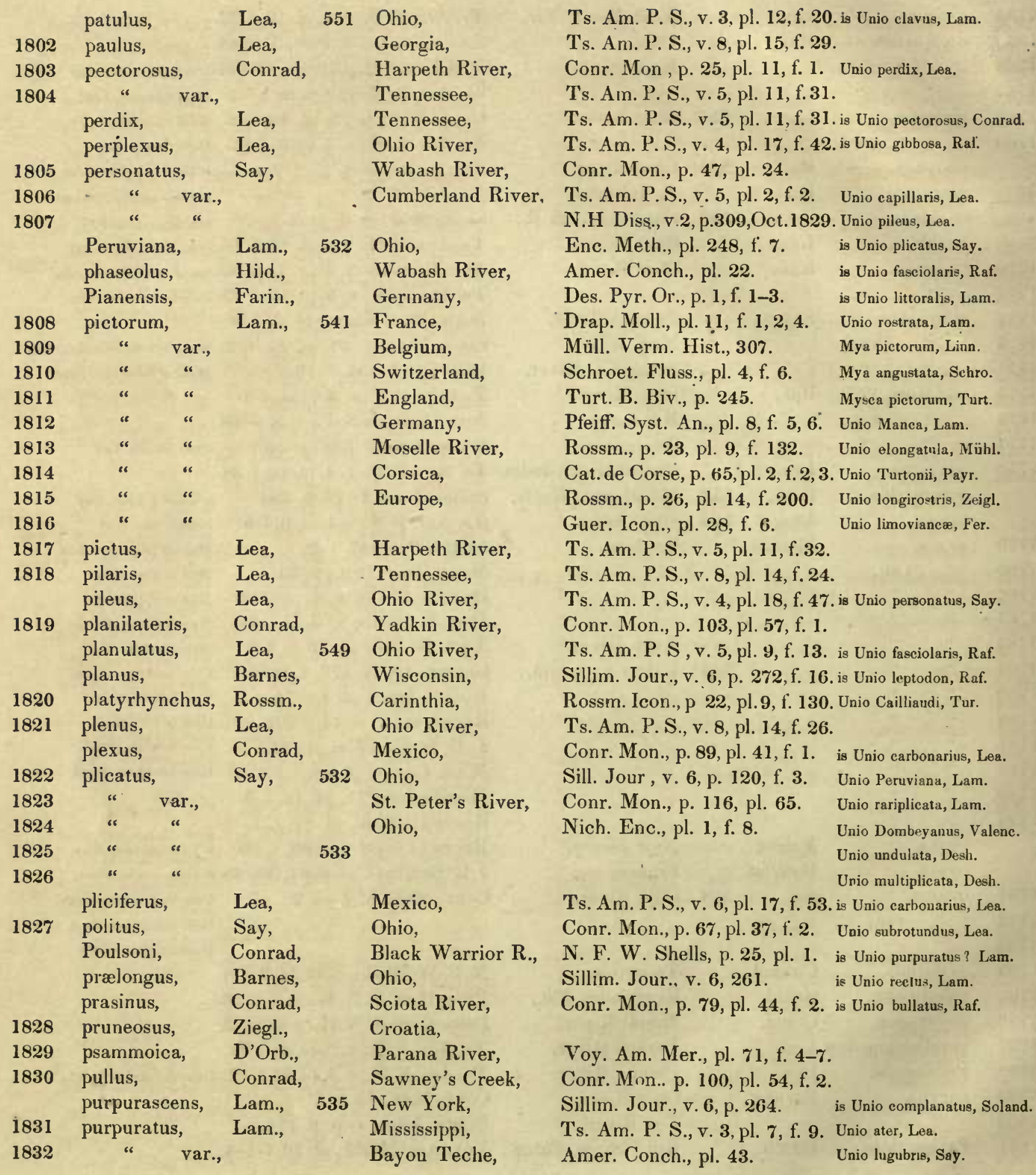






1833

1831

1835

1836

1837

1838

1839

1840

1841

1842

1843

1844

1845

1846

847

1848

1849

1850

1851

1852

1853

1854

1855

1856

1857

1858

1859

1860

1861

1862

1863

1864 purpuratus, var.,

purpureus, Say,

purpuriatus, Say,

pustulatus, Lea,

pustulosus, Lea,

pyramidatus, Lea,

quadrulus, Raf.,

" var.,

"6 "6

"

" "

"6 4

" "

radiatus, Lam.,

" var.,

" "

Rajahensis,

Rangianus,

rariplicata,

rarisulcata,

Raveneli,

Ravenelianus,

rectus,

" var.,

" "

reflexus,

" var.,

reniformis,

Requienii,

retusus,

" val.,

"6

rhomboidea, Schr.,

lhombula,

ridibundus,

Lea,

Lea,

Lam.,

Lam.,

Conrad,

535 Virginia,

Wisconsin,

544 Potomac,

Calcutta,

Ohio River,

533 St. Peter's River,

534

Lake Champlain,

Wateree Canal,

Frenel Broad River,

Lam.,

Raf.,

Schmidt,

Mich.,

Lam.,

riparius,

Lam.,

Say,

Lea,

" var.,

robustus, Parr.,

rostratus, Valen.,

rostratus, Studer.,

rostratus, Lam.,

rotundatus, Lam.,

$$
\text { " var., }
$$

537

534

Ohio River,

Lake Erie,

Detroit River,

Alabama River,

Ohio River,

Carinthia,

Rhone River,

Ohio River,

Tennessee,

Wabash River,

France,

536 Iudson River,

Cumberland River,

Europe,

Roanoke River,

Styria,

New York,

England,

540 France,

538 Bayou Teche,

546 Louisiana,
N. F. W. Shells, p. 25, pl. 1. Unio Poulsoni? Conrad.

Unio ventricosus, Soland.

Nich. Enc. Amer., pl. 3, f. 1. is Unio complanatus, Soland.

Desc. of New Shells, p. 6. Unio Medellinus, Lea. -

Ts. Am. P. S., v. 4, pl. 7, f. 9. is Unio nodulatus, Raf.

Ts. Am. P. S., v. 4, pl. 7, f. 7. is Unio bullatus, Raf.

T's. Am. P. S., v. 4, pl. 16, f. 39. is Unio Mytiloides, Raf,

Conr. Mon., p. 112, pl. 62 . Unio asperrimus, Lea.

Pouls. Transl. Raf., p. 42. Obliquaria quadrula, Raf.

Amer. Conch., pl. 53.

Unio rugosus, Barnes.

Ts. Am. P. S., v. 3, pl. 6, f. 8. Unio lachrymosus, Lea.

Chenu C. Ill., pl. 15, f. 6.

Chenu C. Ill., pl. 9, f. 1.

Ts. Am. P. S., v. 4, pl. 5, f. 3.

Conr. Mon., p. 24, pl. 10, f. 2. Unio Virginiana, Lam.

Sillim. Jour., v. 6, p. 265. Mya radiata, Gmel.

Wood's Supp., pl. 1, f. $2 . \quad$ Mya oblongata, Wood.

Ts. Am. P. S., v. 8, pl. 23, f. 53.

Ts. Am. P. S., v. 8, pl. 18, f. 56.

Conr. Mon., p. 116, pl. 65.

is Unio plicatus, Say.

Schroet. Einl., 2, p. 458.

is Unio eomplanatus, Soland.

N. F. W. Shells, p. 39, pl. 6, f. 4. is Unio Watercensis, Lea.

r, 'Ts. Am. P. S., v. 5, pl. 3, f. 5. Unio rudis, Conrad.

Conr. Mon., p. 33, pl. 15. Unio latissima, Raf.

Sillim. Jour., v. 6, pl. 14, f. 11 . Unio prælongus, Barnes.

Conr. Mon., p. 53, pl. 29, f. 1 . Unio Sageri, Conrad.

Conr. Mon., p. 7, pl. 4, f. 1 . Unio cornutus, Baroos.

Sillim. Jour., 6, p. 122, pl. 4, f. 5.

Rossm., p. 31, pl. 15, f. $213 . \quad$ Unio batavus, Lans.

Mich. Supp., pl. 16, f. 24 . Unio pictorum, Lam.

Conr. Mon., p. 19, pl. 8.

Reeve C. Syst., pl. 88, f. 6. Unio torsa, Raf.

Cuv. R. An. Desh., pl. 92, f. 1. Unio oblusa, Say, Desh.

Schroet. Fluss., pl. 2, f. $3 . \quad$ is Unio littoralis, Lam.

Delessert, pl. 12, f. 8.

is Unio complanalus, Sol.

Amer. Conch., pl. 5.

is Unio obliquatus, Raf.

Wood's Gen. C., pl. 19, f. 1, 2.

Ts. Am. P.S., 6, p. 27, pl. 8, f. 21.

Chenu C. Ill., pl. 20, f. 6.

Cuv. R. An., pl. 92, f. $2 . \quad$ is Unio nasulus, Say.

Lister C., pl. 147, f. $2 . \quad$ is Unio ovalis, Flem.

Mich. Supp., p. 108, pl. 16, f. 25. is Unio pictorum, Lam.

Ts. Am. P. S., 5, p. 30, pl. 2, f. 3. Unio subglobasus, Lea.

Amer. Conch., pl. 34.

Unio suborbiculata, Lam.

Jr. Transyl. Univ., v. 4, p. 526. Unio glebulus, Say. 


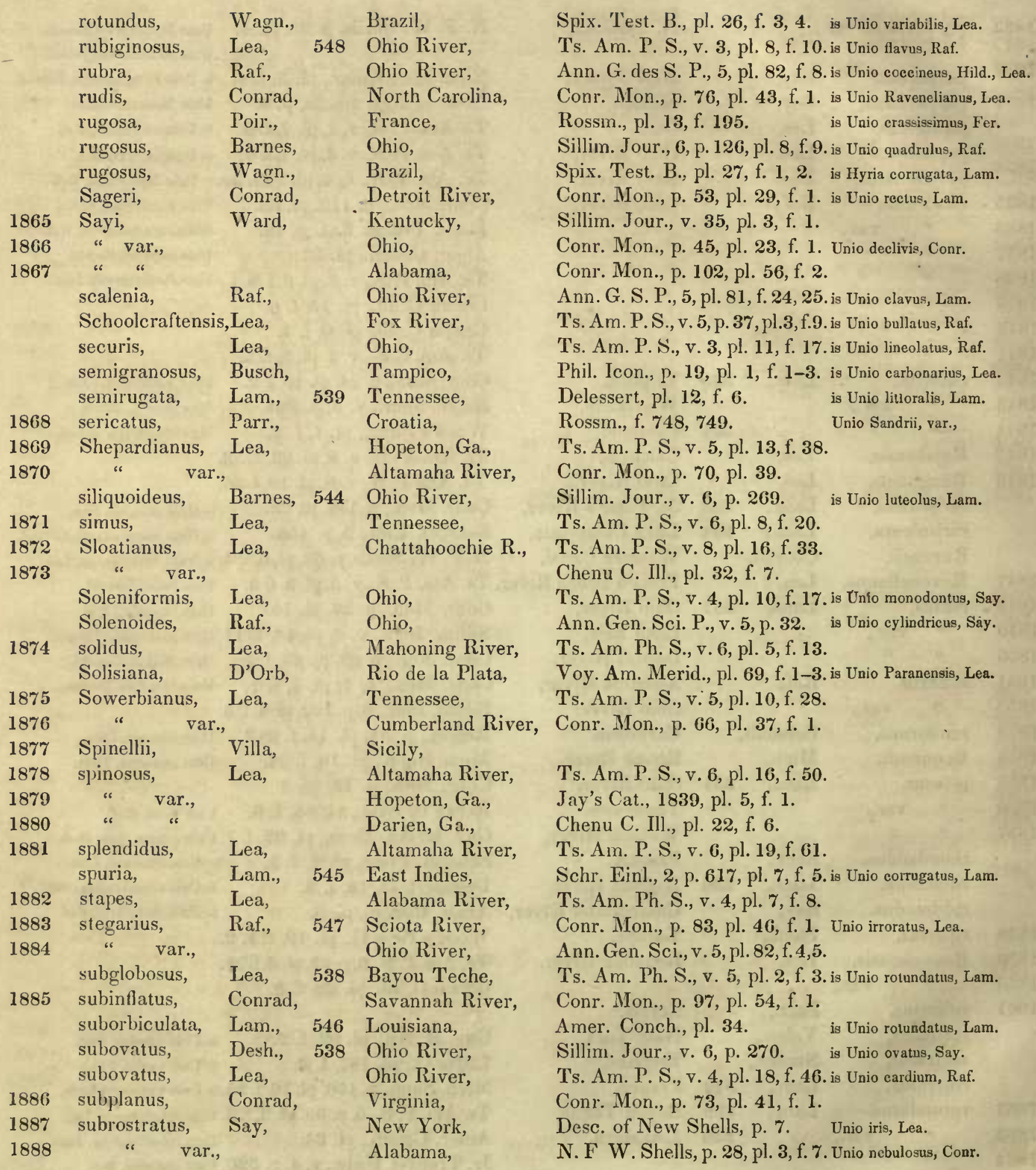








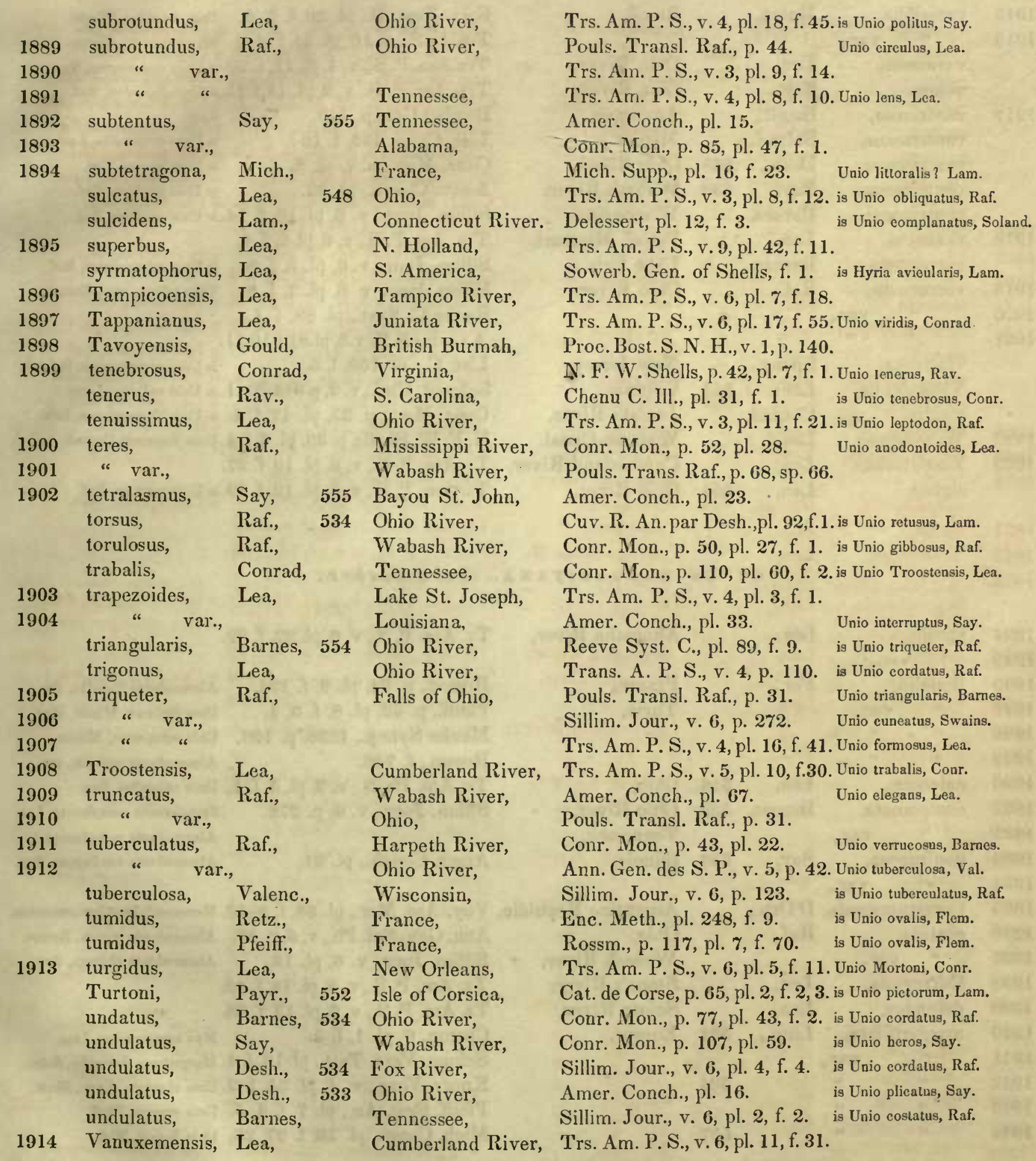




\begin{tabular}{|c|c|c|c|c|c|}
\hline \multirow{4}{*}{$\begin{array}{l}1915 \\
1916\end{array}$} & variabilis, & \multicolumn{2}{|l|}{ Maton, } & \multirow[t]{2}{*}{ Brazil, } & \multirow{3}{*}{$\begin{array}{l}\text { Spix. 'Test. B., pl. } 26, \text { f. } 3,4 \text {. Unio rolnndus, Wagn. } \\
\text { Trs. L. S., v. } 10 \text {, pl. 24, f. 4-7. Mya variabilis, Maton. } \\
\text { Trs.Am. P.S., v. 4, pl. 11, f. } 20 \text {. is Unio cicatricosus, Say. }\end{array}$} \\
\hline & var., & & & & \\
\hline & varicosus, & Lea, & & Ohio River, & \\
\hline & velum, & Say, & & Ohio & Trs. Am. P. S., v. 3, pl. 11, f.21. is Unio Ieplodon, Raf. \\
\hline 1917 & ventricosa, & Bouill., & & Auve & Bouillet Cat., p. 149. \\
\hline & ventricosus, & Soland., & & Miss & List. C., pl. 155, f. $10 ?$ \\
\hline & ricosus, & Desh., & 538 & Ohio R & Conr. Mon., p. 4, pl. 2. \\
\hline & icosus, & Say, & 538 & iver, & Amer. Conch., pl. 32. \\
\hline & ventricosus, & Barnes, & & & Sillim. Jour., v. 6, pl. 13, f. 14. is Unio eardium, Raf. \\
\hline 1918 & $\begin{array}{l}\text { venustus, } \\
\text { verrucosus, }\end{array}$ & $\begin{array}{l}\text { Lea, } \\
\text { Barnes, }\end{array}$ & & $\begin{array}{l}\text { Missouri, } \\
\text { Erie, }\end{array}$ & Trs. Am. P. S., v. 6, pl. 2, f. 4 . \\
\hline 1919 & verrucosus, & Raf., & & Ohi & Sillim. Jour., v. 6 , pl. 7 , f. $8 . \quad$ Unio tubcreulatus, Barnes. \\
\hline 920 & var., & & - & & pl. 88 , f. 1. \\
\hline 1921 & “ & & & & A.G. des S.P., v.5, pl.81,f.10,11. \\
\hline & violaceus, & Spe & & Jersey, & is Unio complanatus, Soland. \\
\hline & anus, & Lam., & 544 & Virginia, & Schroet. Einl., У. 2, p.614,No.1. is Unio radiatus, Lam. \\
\hline 0 & & Conrad, & & Jun & 1. is Unio Tappanianus, Le \\
\hline 922 & Watereensis, & Lea, & & Wateree Canal, & N. F. W. Shells, p. 39, pl. 6, f.4. Unio Raveneli, Conrad. \\
\hline 19 & $\begin{array}{l}\text { Zieglerianus, } \\
\text { zigzag, }\end{array}$ & $\begin{array}{l}\text { Lea, } \\
\text { Lea, }\end{array}$ & & $\begin{array}{l}\text { Cumberland R } \\
\text { Ohio River, }\end{array}$ & $\begin{array}{l}\text { Ts. Am. P. S., v. 6, pl. 10, f. } 27 \text {. } \\
\text { Ts. Am. P. S., v. 3, pl. 12, f. 19. is Unio nervosa, Raf. }\end{array}$ \\
\hline
\end{tabular}

GENUS MARGARITANA. Schumacher.

\begin{tabular}{|c|c|c|c|c|c|c|}
\hline $\begin{array}{l}1924 \\
1925\end{array}$ & $\begin{array}{l}\text { arcula, } \\
\text { "var., }\end{array}$ & Lea, & & Altamaha River, & Ts. Am. P. S., v. 6, pl. 22, f. 69. & \\
\hline 1926 & Bonellii, & Fer., & & France, & Rossm., p. 24, pl. 9 , f. 134. & Alasmodonia Bonellii, Fer. \\
\hline 1927 & var., & & & Turin, & Pfeiff., p. 32, pl. 8, f. 3,4 . & Unio depressa, MühIf. Meg. \\
\hline 1928 & " . " & & & & Menke Synop., 1830, p. 106. & Unio compressa, Manke. \\
\hline 1929 & " & & & & Rossm., f. 746. & Alasmodonta uniopsis, var. \\
\hline 1930 & calceola, & Lea, & 546 & Ohio, & Ts. Am. Ph. S., v. 3, pl, 3, f. 1. & Unio caleeolus, Lea. \\
\hline 1931 & complanata, & Barnes, & 559 & Ohio River, & Sillim. Jour., v. 6, p. 278. & Symphynota complanata, Lea. \\
\hline 1932 & var., & & & Tennessee, & & Alasmodonta complanata. \\
\hline 1933 & confragosa, & Say, & 553 & Illinois, & Amer. Conch., pl. 21. & Alasmodonta confragosa, Say. \\
\hline 1934 & " var., & & & & & \\
\hline 1935 & Corrientesensis, & $\mathrm{D}^{\prime}$ & & Argentine Republic, & Voy. Am. Mer., pl. 68, f. 8-10. & Monoc ondyløea Corrientesensis. \\
\hline 1936 & costata, & af., & & Ohic & Ann. Gen. Sci. Ph., v. 5. p. 49. & Alasmodonta rugosa, Barnes. \\
\hline 1937 & var., & & & Wabash River, & Sillim. Jour., v. 6, pl. 13. & Alasmodonta abducta, Say. \\
\hline 1938 & Curreyana, & Lea, & & Stone's River, & Ts. Am. P. S., v. 8, pl. 18, f. 40. & \\
\hline 1939 & deltoidea, & Lea & & Sciota River, & Ts. Am. P. S., v. 6, pl. 13, f. 38. & \\
\hline 1940 & margaritifera, & Linn., & 531 & Europe, & Chemn. 6, pl. 1, f. 5. & Mya margaritifera, Liun. \\
\hline 1941 & var., & & & & Ters. Test., $\mathrm{P}$ & - Margaritana fluviatilis, Sohu. \\
\hline 1942 & “ & & 531 & & Knorr. Verg., v. 4, pl. 25, f. 2. & Unio elongala, Lam., Mich. \\
\hline 1943 & " & & & & Pfeiff. Syst. An., pl. 7, f. 4, 5. & Unio sinuata, Pfeiff. \\
\hline 1944 & " & & 557. & & Mich. Comp., pl. 16, f. 27, 28. & Unio Roissii, Mich. \\
\hline
\end{tabular}








\begin{tabular}{|c|c|c|}
\hline 1946 & $\underset{\text { margaritifera, } v}{ }$ & var., \\
\hline 1947 & " & " \\
\hline 48 & " & “ \\
\hline 949 & marginata, & Say, \\
\hline 50 & var., & \\
\hline 51 & " & \\
\hline 52 & " & \\
\hline 53 & " & \\
\hline 54 & “ & \\
\hline 955 & $\begin{array}{l}\text { Raveneliana, } \\
\text { rugosa, }\end{array}$ & $\begin{array}{l}\text { Lea, } \\
\text { Barnes, }\end{array}$ \\
\hline 56 & undulata, & Say, \\
\hline & " var., & 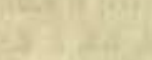 \\
\hline bs & " " & \\
\hline & " " & \\
\hline
\end{tabular}

1961 Vondeınbuschiana, Lea,
England,

France,

England,

Hudson River,

New York,

New Jersey,

545 Lake Erie,

543 Schuylkill River,

Ohio River,

Swananoe River,

Fox River,

New York,

Virginia,

New Jersey,

Java,
Turt. Man., p. 19, pl. 2, f. 9. Unio margaritiferus, Pfeiff.

Drap., pl. 10, f. 17-19. Unio rivalis, Ziegl.

Flem. B. Anim.,p. 417, No. 429. Alasmodonta margaritiferum.

Sillim. Jour., v. 6, pl. 12, f. 20. Alasmodonta arcuata, Barnes.

Jour. Ac. N. S., v. 1, p. 459 . Alasmodonta marginata, Say.

Alasmodonta truncata, Say.

Unio cariosa, Lam., var. ?

Unio varicosa, Lam.

Unio calceolus, Say.

Wood's Supp., pl. 1, f. 7 . Mya regulosa, Wood.

Ts. Am. P. S., v. 5, pl. 17, f. 50.

Sillim. Jour., v. 6, pl 13, f. 21 . is Margaritana costata, Raf.

Nich. Enc. Am., pl. 3, f. 3. Alasmodonta undulata, Say.

Say in Pamph., 1840, p. 9. Alasmodonta seulptilis, Say.

Wood's Supp., pl. 1, f. 5. Mya undulata, Wood.

Unio hians, Valenc.

Sowb. Gen., f. 3.

Unio glabratus, Sọwb.

Ts. Am. P.S., v. 8, pl. 18, f. 39.

GENUS DIP\$A L. Leach.

$\begin{array}{llllll}1962 & \text { discoideus, } & \text { Lea, } & & \text { China, } \\ 1963 & \text { " var., } & & & \\ 1964 & \text { " } & \text { " } & & & \\ 1965 & \text { plicatus, } & \text { Leach, } & \text { Canton, } \\ 1966 & \text { " } & \text { var., } & & 558 & \\ 1967 & \text { " } & \text { " } & & & \\ 1968 & \text { " } & & & \\ 1969 & \text { " } & & & \\ 1970 & \text { " } & & & \end{array}$

G ENUS II RIA. Lamarck.

Ts. Am. P. S., v. 5, pl. 11, f. 33. Symphynota discoidea, Lca.

Grif. Cuv., pl. 24, f. 2 . Unio tenuis, Gray.

Grif. Cuv., pl, 24, f. $2 . \quad$ Anodonta tenuis, Gray.

Ts. Am. P. S., v. 3, pl. 14, f. 24. Symphynota bi-alata, Lea.

Unio bi-alata, Desh.

Unio bilineatus, Bens.

Mytilus dubius, Gmel.

Anodon alatus, Sowb.

Blain Mal., pl. 66, f. 3.

Anodonta dipsas, Blain.

\begin{tabular}{|c|c|c|c|c|}
\hline 1971 & avicularis, & \multirow[t]{4}{*}{ Lam., } & \multirow[t]{3}{*}{561} & \multirow{3}{*}{$\begin{array}{l}\text { Amazon River, } \\
\text { South America, } \\
\text { Guiana, }\end{array}$} \\
\hline 1972 & var., & & & \\
\hline 1973 & $"$ & & & \\
\hline 1974 & " & & & \\
\hline 1975 & Browniana, & Lea, & & Amazon River, \\
\hline 976 & corrugata, & Lam., & 562 & South America, \\
\hline 1977 & var., & & & \\
\hline 1978 & " & & & Solimoês River, \\
\hline 1979 & " & & & Guiana, \\
\hline 880 & " & & & \\
\hline
\end{tabular}

Delessert, pl. 12, f. 9.

Swains. Exot. C., pl. 24. Hyria elongata, Swains.

Icon. du R. Anim., pl. 28, f. 8. Unio caudatus, Wagn.

Spix. Text. B., p. 35, pl. 27, f. 1,2.

Ts. Am. P. S., v. 6, pl. 24, f. 116.

Enc. Meth., pl. 247, f. $2 . \quad$ Unio corrugatus, Blain.

Wood's Supp., pl. 1, f. $12 . \quad$ Mya angulata, Wood.

Sch. Ess. Class. Coq., pl. 11, f. 3. Paxyodon ponderosus, Schum. Spix. 'T. B., p. 35, pl. 29, f. 1, 2. Triplodon rugosus, Spix.

Unio rugosus, Wagn. 


$\begin{array}{lcc}1981 & \text { syrmatophora, Sowb., } \\ 1982 & \text { " } & \text { var., } \\ 1983 & \text { " } & \text { " } \\ 1984 & \text { " } & \text { " } \\ 1985 & \text { " } & \text { " } \\ 1986 & \text { " } & \text { " } \\ 1987 & \text { " } & \text { " }\end{array}$

$\begin{array}{lll}\text { Amazon River, } & \text { Sowb. Gen., f. 1. } & \text { Mya syrmatophora, Linn. } \\ & \text { Ts. Am. Ph. S., v. 6, p. 121. } & \text { Unio syrnatophora, Lea. } \\ & \text { List. C., pl. 160, f. 16. } & \text { Hyria avicularis, Desh. } \\ & \text { Schum. Vers., pl. 11, f. 2, a, b. Prisodon obliquus, Schum. } \\ & \text { Solimoês River, } & \text { Spix. Test. B., pl. 27, f. 1, 2. Diplodon furcatum, Spix. } \\ & \text { Gron. Zooph., p. 260, pl. 18, f. 1. Mya syrmalophora, Gron. } \\ & \text { Wood's Ind., pl. 2, f. 36. } & \text { Unio caudatus, Wagn. } \\ & \text { Cuv. R. An. Desh., pl. 92, f. 3. }\end{array}$

GENUS IRIDINA. Lamarck.

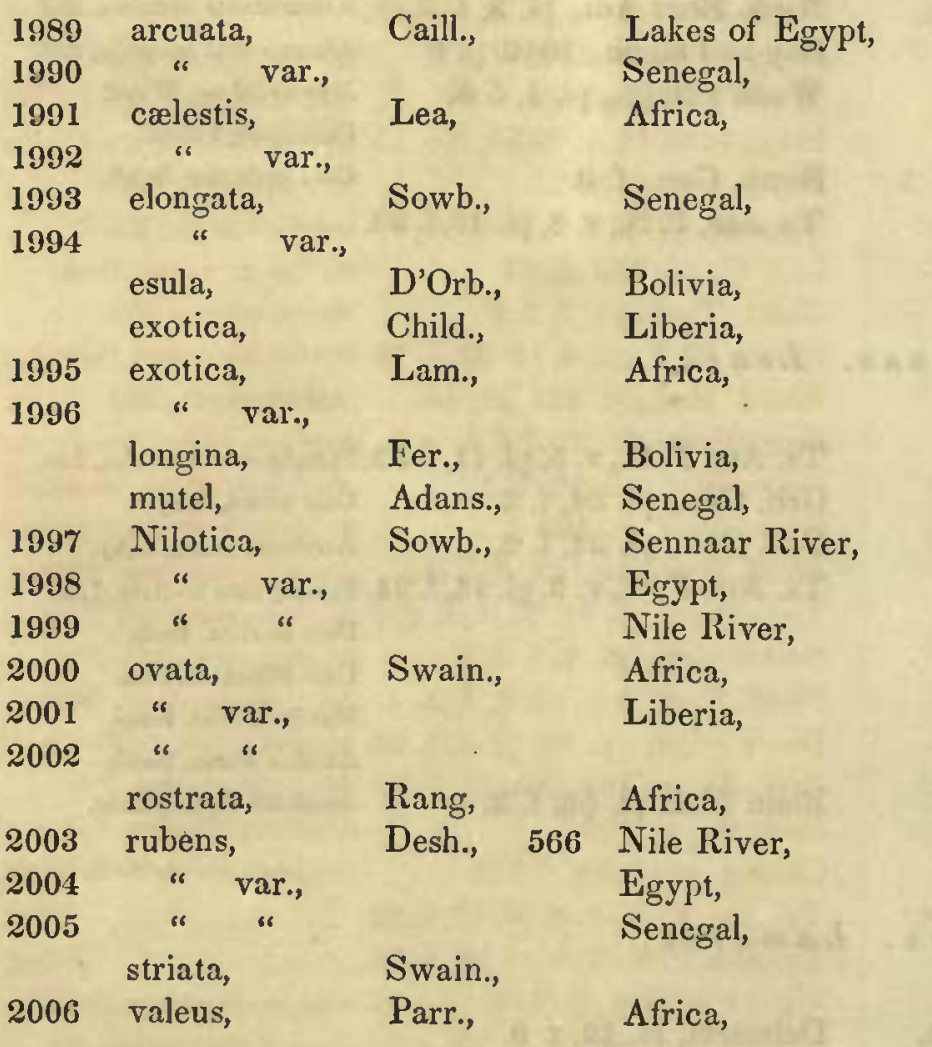

Voy. à Méroé, v. 2, pl. 61, f. 4,5.

Pot. et Mich., v. 2, pl. 55, f. 4.

Ts. Am. P. S., v. 6, pl. 22, f. 70. Spatha cælestis, Lea.
Reeve C. Syst., pl. 92. Iridina rostrata? Rang. Spatha elongata, Lea.

Iridina exotica, Desh. Chenu, pl. 1, f. $2 . \quad$ is Iridina ovata, Swains. Enc. Meth., pl. 204, bis. f. 1. Iridina striala, Swains. Blain. Mal., pl. 66, f. 3. Anodonta exotica, Blain. Voy. Am. Merid., pl. 67. is Mycetopus siliquosa, D'Orb. Adans. Sen., p. 234, pl. 17, f. 21. is Iridina Nilotica, Sowb. Zool. Jour., v. 1, p. 53, pl. 2. Iridina mutel ? Rang. Wood's Supp., pl. 2, f. 1. Iridina Oudniæi, Kœenig. Voy. à Méroé, v. 2 , pl. 60, f. 11. Iridina mutel, Adans. Reeve C. Syst., pl. 93, f. 1, 2. Pleiodon McMurtrei, Conr. Wood's Supp., pl. 2, f. $9 . \quad$ Iridina exotica, Child. Jour. Ac. N. S., v. 7, pl. 13. Pot. et Mich., v. 2, pl. 56, f. 1. is Iridina cælestis, Lea. Enc. Meth., pl. 201, f. 1. Anodonta rubens, Lam. Voy. à Méroé, v. 2, pl. 60, f. 12. Anodon Clappertoni, Den. Cuv. R. An. par Desh., 91, f. 1. White, var. Chenu C. Ill., pl. 1, f. $1 . \quad$ is Iridina exotica, Lam.

GENUS A NODONTA. Bruguière.

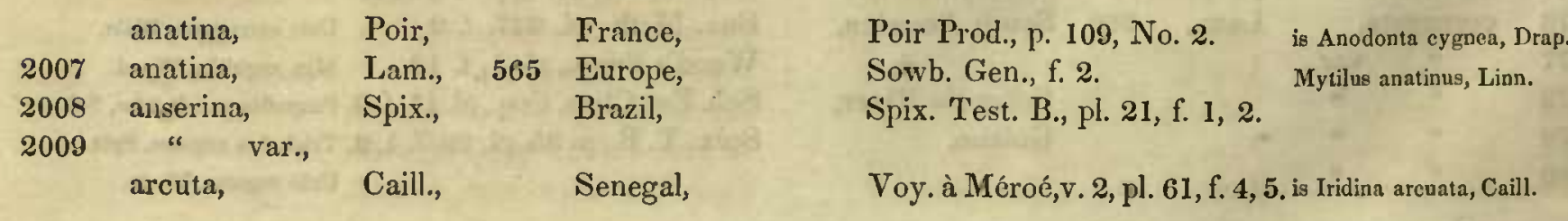





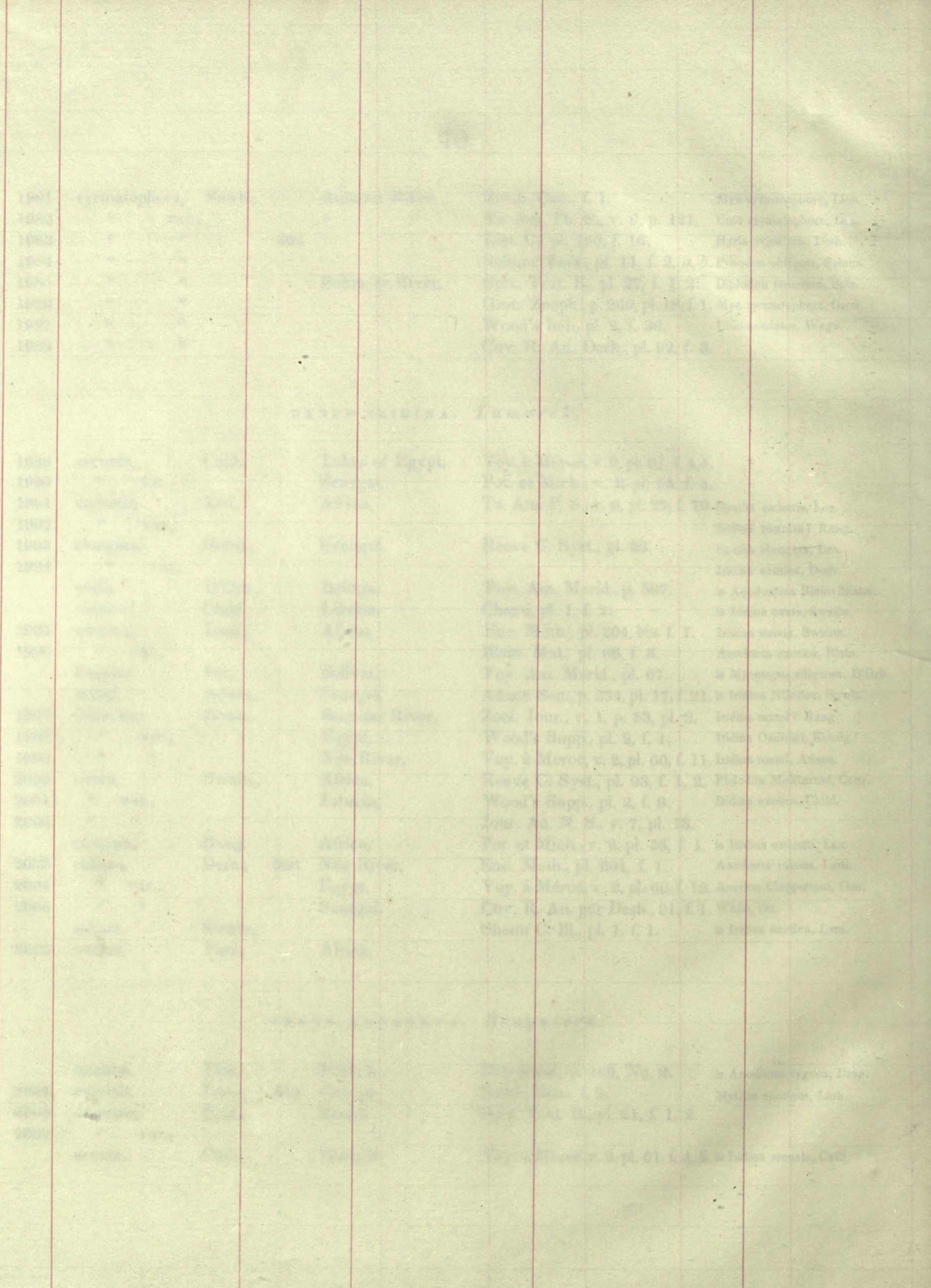






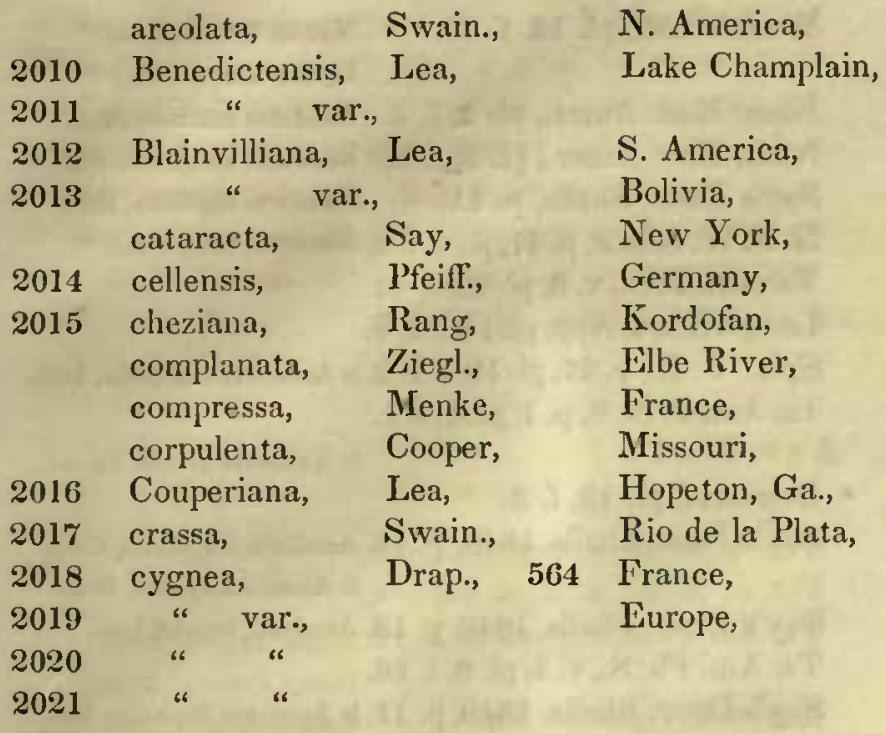

566

\begin{tabular}{|c|c|c|}
\hline 022 & cylindracea, & Lea, \\
\hline 2023 & $\begin{array}{l}\text { decora, } \\
\text { dentiens, }\end{array}$ & $\begin{array}{l}\text { Lea, } \\
\text { Menke, }\end{array}$ \\
\hline 2024 & edentula, & Say, \\
\hline 2025 & " var., & \\
\hline 2026 & elongata, & Swains., \\
\hline 2027 & ensiformis, & Spix, \\
\hline 2028 & excurvata, & De Kay, \\
\hline 2029 & exilis, & Lea, \\
\hline & exotica, & Lam., \\
\hline 2030 & exulcerata, & Villa, \\
\hline 2031 & ferruginea, & Lea, \\
\hline 032 & Ferussaciana, & Lea, \\
\hline
\end{tabular}
France, Ohio, Wabash River, Brazil, Rio Negro, Passaic River,

Peru, Italy, Indiana, Cincinnati,
Swain. Z. Ill., 2d ser., pl. 18. is Anodonta edentula, Lea. Trs. Am. P.S., v. 5, pl. 16, f. 48. Symphynota Benedictensis, Lea.

Trs. Am. P. S., v. 5, pl. 12, f. 35. Iridina esula, D'Orb.

Voy. Am. Merid., p. 597.

Nich. Enc. Am., pl. 3, f. $4 . \quad$ is Anodonta fluviatilis, Lea. Pfeiff. Moll., pl. 6, f. 1-4.

Rossm., p. 112, pl. 3, f. 68.

Menke Synop., p. $106 . \quad$ is Anodonta cygnca, Drap. Say's Des. of Shs. pamph. p. 12. is Anodonta grandis, Say. Trs. Am. P. S., v. 8, pl. 20, f. 46.

Sw. Z. Ill., 1st ser., v. 3, pl. 167.

Drap. Moll., p. 134, pl. 11. Mytilus cygnens, Gmel. Wood Index, pl. 12, f. 32. Mytilus stagnalis, Gmel. Linn. 1158, G., p. 3355, No. 15. Mytilus fluviatilis, Gmol. Dillw. Cat., p. 317. Mytilus fucatus, Dillw.

Schroet., pl. 2, f. 1. Mytilus Zellensis, Gmel. List. C., pl. 156, f. 11 Mytilus Avonensis, Mont. Gault. Test., pl. 7, f. F. Mytilus radiatus, Mühl. Pennt. B. T., v. 4, pl. 67, f. 78. Mylilus incrassatus, Shep. Brard. Hist., p. 234, pl. 10. Mytilus macula, Shep. Enc. Meth., pl. 202, f. 1, $a, b$. Anodonta sulcata, Lam. Blain. Mal., pl. 66, f. $1 . \quad$ Anodonta dentiens, Menke. Drap. Moll., p. $133 . \quad$ Anodonta variabilis, Drap. Pfeiff. Syst. An., pl. 6, f. 4. Anodonta ventricosa, Pfeiff. Turt. Man., p. 17, pl. 1, f. 8. Anodonta paludosus, Turt. Sowb. Gen., pl. 1, f. 1, 2. Anodonta grossa, Ziegl. Pfeiff. Syst. An., pl. 6, f. 2. Anodonta compressa, Menke. Brook's Intr., p. 86, pl. 4, f. 49. Anodonla obvoluta, Ziegl. Poir. Pr., p. 108, No. 1. Anodontites cygnea, Poir. Poir. Pr., p. $109 . \quad$ Anodontites anatina, Poir. Chemn. 8, pl. 86, f. $763 . \quad$ Anodonta proboscidalis, Ziegl. Medellin River, Ts. Am. P. S., v. 6, pl. 13, f. 40. Chilicothe, Ts. Am. P. S., v. 6, pl. 20, f. 63.
Menke Synop., p. $106 . \quad$ is Anodonta cygnea, Drap. Say's Desc. Shells, p. $10 . \quad$ Alasmodonta edentula, Say.

Swains. Z. Ill., 2d ser., pl. 18. Alasmodonta areolatus, Swain. Swains. Z. Ill., 1st ser., 3, pl.176.

Voy. Am. Merid., pl. 79, f. 10.

N. H. N. York, pl. 17, f. 233. Anodonta fluviatilis? Lea. Ts. Am. P. S., v. 6, pl. 22, f. 68.

Delessert, pl. 13, f. $1 . \quad$ is Anodonta trapezialis, Lam.

Porro. Mal., p. 111, pl. 2, f. 12.

Ts. Am. P. S., v. 8, pl. 19, f. 43.

Ts. Am. P. S., v. 5, pl. 6, f. 15. 


\begin{tabular}{|c|c|c|c|c|c|}
\hline 033 & $\begin{array}{l}\text { fluviatilis, } \\
\text { "s var., }\end{array}$ & Lea, & & $\begin{array}{l}\text { Hudson River, } \\
\text { New Jersey, }\end{array}$ & Wood's Ind., pl. 12, f. 35. \\
\hline 035 & “ & & 566 & New & Nich. Enc. Amer., pl. 3, f. 4. Anodonta cataracta, Say. \\
\hline 036 & “ & & & etts, & Nich. Enc. Amer., pl. 3, f. 3. Anodonta marginata, Say. \\
\hline 037 & " & . & & $\mathrm{C}$ & Say's Desc. Shells, p. 11. \\
\hline 038 & " & & & & 7, f. 2 . Anodonta teres, Conr. \\
\hline 2039 & Footiana, & Lea, & & & 44. \\
\hline 40 & ssa, & Say, & & a, & 4. \\
\hline 2041 & $\begin{array}{l}\text { gigantea, } \\
\text { gigantea, }\end{array}$ & Lea, & & ibson, & $\begin{array}{l}\text { Spix. T. B., p. } 27 \text {, p]. 19, f. 1, } 2 . \text { is Anodo } \\
\text { Ts. Am. P.S., 6, p. 1, pl. 1, f. } 1 \text {. }\end{array}$ \\
\hline & & & 569 & & is Anodonta glauca, Valene \\
\hline 042 & $\mathrm{~g}$ & c., & 569 & & \\
\hline 043 & $\begin{array}{l}\text { grandis, } \\
\text { grossa, }\end{array}$ & Ziegl., & & & $\begin{array}{l}\text { 12. } \mathrm{Ar} \\
\text { is }\end{array}$ \\
\hline 2044 & $\begin{array}{l}\text { imbecillis, } \\
\text { " var., }\end{array}$ & Say & & iver, & $\begin{array}{l}\text { p. 13. Anodonta incerta, Lea. } \\
\text { f. } 16 \text {. }\end{array}$ \\
\hline & $\begin{array}{l}\text { implicata, } \\
\text { incerta, }\end{array}$ & $\begin{array}{l}\text { Say, } \\
\text { Lea, }\end{array}$ & 508 & tts, & $\begin{array}{l}\text { 's Desc. Shells, 1840, p. 11. is Anodont } \\
\text { Am. Ph. S., v. 5, pl. 6, f. 16. is Anodont }\end{array}$ \\
\hline 2046 & $\begin{array}{l}\text { intermedia, } \\
\text { lata, }\end{array}$ & $\begin{array}{l}\text { Lam., } \\
\text { Raf., }\end{array}$ & 568 & $\mathrm{~F}$ & 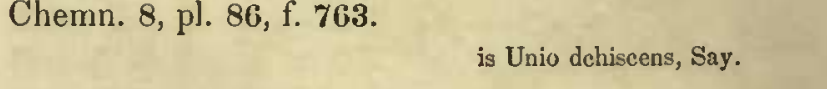 \\
\hline 047 & latissima, & Kokeil, & & & \\
\hline 048 & lato-marginata, & Lea, & & & S., v. 5, \\
\hline $\begin{array}{l}049 \\
050\end{array}$ & $\begin{array}{cc}\text { " } & \text { var., } \\
\text { " } & \text { “ }\end{array}$ & & & a River, & $\begin{array}{l}\text { 1. An } \\
2-4 . A n\end{array}$ \\
\hline 2051 & “ & & & & 1. \\
\hline 2052 & leprosa, & Parr., & & & \\
\hline 2053 & lii & $\mathbf{P}$ & w & & \\
\hline $\begin{array}{l}2054 \\
2055\end{array}$ & $\begin{array}{l}\text { lituratum, } \\
\text { magnifica, } \\
\text { “ var., }\end{array}$ & $\begin{array}{l}\text { Spix, } \\
\text { Lea, }\end{array}$ & & $\begin{array}{l}\mathrm{B} \\
\mathrm{C}\end{array}$ & Am. Ph. S., v. 5, pl. \\
\hline 2056 & $\begin{array}{l}\text { marginata, } \\
\text { minima, }\end{array}$ & $\begin{array}{l}\text { Say, } \\
\text { Mill., }\end{array}$ & & $\begin{array}{l}\mathrm{N} \\
\mathrm{N}\end{array}$ & $\begin{array}{l}\text { 3. is } \\
\text { f.2. }\end{array}$ \\
\hline 2057 & Mon & Lea & & erica, & 55. \\
\hline 2058 & ensis, & Le & & & 66. \\
\hline 2059 & Nuttalliana, & Lea, & & V & 62. \\
\hline 2060 & $\begin{array}{l}\text { obtusa, } \\
\text { obvoluta, }\end{array}$ & $\begin{array}{l}\text { Spix, } \\
\text { Ziegl., }\end{array}$ & & & $\begin{array}{l}\text { 4. Anodonta liturat } \\
\text { is Anodonta eygt }\end{array}$ \\
\hline $\begin{array}{l}2061 \\
2062\end{array}$ & $\begin{array}{l}\text { Oregonensis, } \\
\text { ovata, } \\
\text { ovatus, } \\
\text { paludosus, }\end{array}$ & $\begin{array}{l}\text { Lea, } \\
\text { Lea, } \\
\text { Srvains., } \\
\text { Turt., }\end{array}$ & & $\begin{array}{l}\text { amat River, } \\
\text { tta, }\end{array}$ & 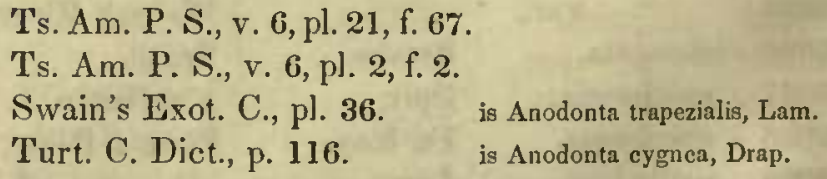 \\
\hline 2063 & nica, & $\mathrm{La}$ & 570 & $P$ & 70, f. $1-4$. Un \\
\hline & $\begin{array}{l}\text { pavonia, } \\
\text { penicillatus, }\end{array}$ & $\begin{array}{l}\text { Lea, } \\
\text { Gra! }\end{array}$ & & eaver & $\begin{array}{l}65 . \\
\text { is Anodonta trapezialis, La }\end{array}$ \\
\hline & $\begin{array}{l}\text { vanica, } \\
\text { na, }\end{array}$ & & 567 & & i. is Ano \\
\hline & piniana, & & & ק & 51 \\
\hline
\end{tabular}








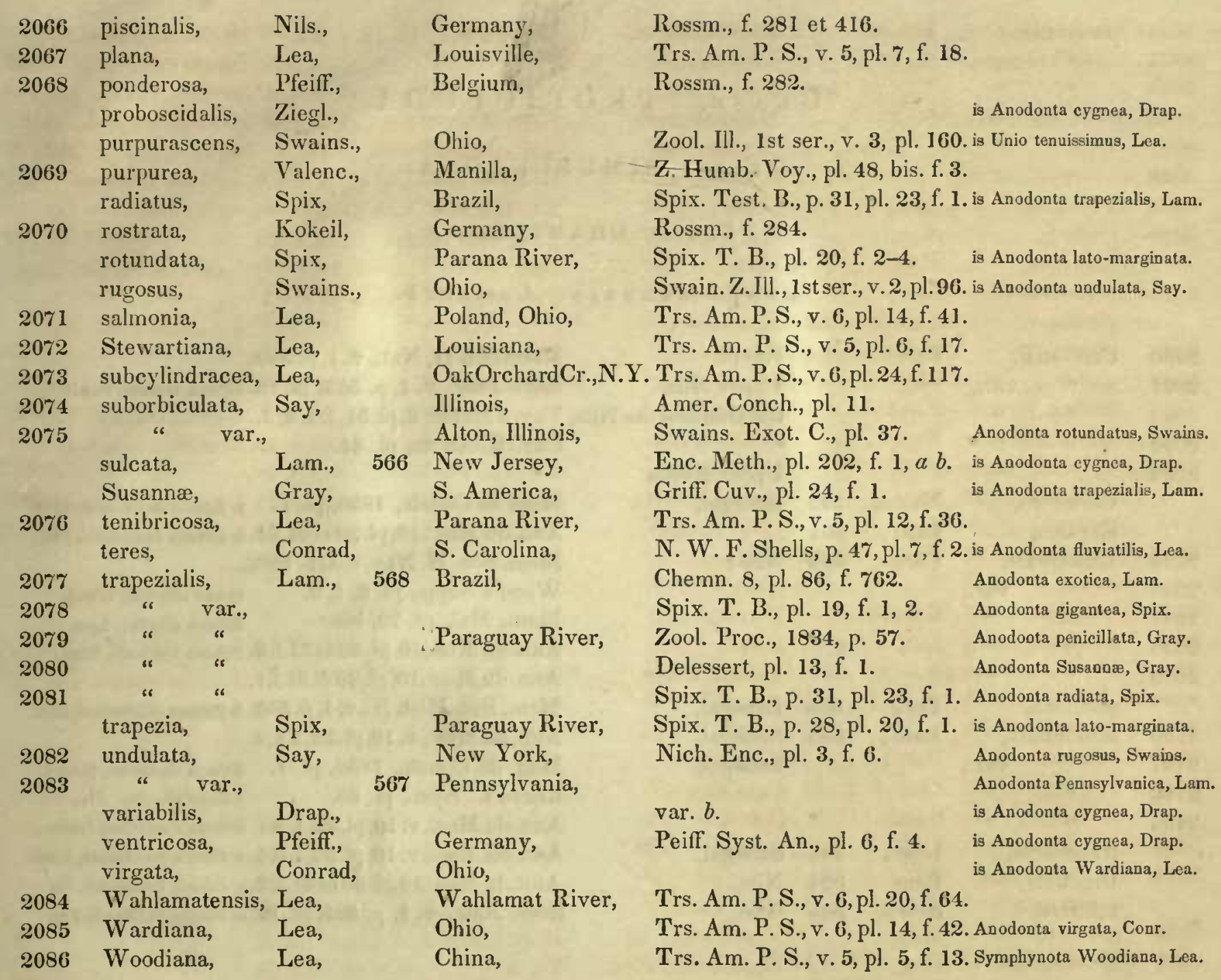

GENU MY E TOPUS. D'Orbigny. 
CLASA. TROPIOPODA.

ORDER. BIMUSCULOSA.

FAMII CHAMACEA.

G ENUS ETHERIA. Lamarck.

\begin{tabular}{|c|c|c|c|c|c|}
\hline \multirow{5}{*}{$\begin{array}{l}2090 \\
2091 \\
2092 \\
2093 \\
2094\end{array}$} & Cailliaudi, & \multicolumn{2}{|l|}{ Fer., } & \multirow{3}{*}{$\begin{array}{l}\text { Egypt, } \\
\text { Upper Nile, } \\
\text { Cataracts of the Nile, }\end{array}$} & \multirow{3}{*}{$\begin{array}{l}\text { Mem. S. d'H. Nat., v. 1, p. } 359 . \\
\text { Zool. Jour., v. 1, p. 523, pl. } 19 . \text { Etheria tubifera, Sowb. } \\
\text { Voy. à Méroé, 2, pl.51, f. 1,2, 3. }\end{array}$} \\
\hline & var., & & & & \\
\hline & “ & & & & \\
\hline & “ & & & & Cuv. R. Anim., pl. 84. \\
\hline & “ & & & $\mathrm{U}_{\mathrm{l}}$ & \\
\hline & Carteroni, & & & & Mag. de Conch., 1830, pl. 1. is Etheria semilunata, Lam. \\
\hline 095 & $\begin{array}{l}\text { elliptıca, } \\
\text { Lamarckii, }\end{array}$ & n., & $\begin{array}{l}594 \\
594\end{array}$ & Nil & $\begin{array}{l}29 \text { et } 31, \text { f. } 1 \text {. is Etl } \\
\text { v. } 1, \text { p. } 359 \text {. }\end{array}$ \\
\hline 096 & var., & & & & Wood's Supp., pl. 2, f. $6 . \quad$ Ost \\
\hline 97 & « & & & & Blain. Mal., pl. 70, bis. \\
\hline 98 & " & & & & Ann. du Mus.,10, pl. 30 et 31,f.2. Etheria trigonula, Lam. \\
\hline 99 & " & & & & f.1. \\
\hline & plumbea, & & 595 & Senegal, & Hist. N., v. 1, p. 359. is Etheria semilunata, Lam. \\
\hline 100 & semilunata, & $\mathbf{L}$ & 595 & Sen & $2, f .1,2$. \\
\hline 01 & var & & & & Etheria $\mathbf{C}$ \\
\hline 10 & “ & & & & Etheria plumbea, Fer. \\
\hline & “ & & & & Ann. du Mus., v. 10, pl. 32, f. 3,4. Etheria transversa, Lam. \\
\hline & transversa, & Lam., & 595 & Senegal, & Mus., v. 10, pl. 32, f. 3,4. is Etheria semilunata, Lam. \\
\hline & $\begin{array}{l}\text { trigonula, } \\
\text { tubifera, }\end{array}$ & & 595 & Nile, & $\begin{array}{l}\text { Ann. du Mus.,10,pl. } 30 \text { et } 31 \text {, f.2. is Etheria Lamarckii, Fer. } \\
\text { Zool. Jour., v. } 1 \text {, p. } 523 \text {, pl. 19. is Etheria Cailliaudi, Fer. }\end{array}$ \\
\hline
\end{tabular}

GENUS DICERAS. Lamarck.

2104 Arietina, Lam.

Mt. Salere,

fossil.

GENUS C II MA. Linnaus.

$\begin{array}{lllrl}2105 & \text { arcinella, } & \text { Linn., } & 584 & \text { Florida, } \\ 2106 & \text { " var., } & & & \\ 2107 & \text { calcarata, } & \text { Lam., } & 589 & \begin{array}{l}\text { Grignon, } \\ \text { Real Llejos, }\end{array} \\ 2108 & \begin{array}{l}\text { corrugata, } \\ \text { damæcornis, }\end{array} & \begin{array}{l}\text { Brod., } \\ \text { Lam., }\end{array} & 580 & \begin{array}{l}\text { Indian Seas, } \\ \end{array}\end{array}$

Chemn. 7, pl. 52, f. 522, 523.

Enc. Meth., pl. 107, f. 4, $a, b$.

Seba. Mus., 3, pl. 106, f. 53, 54.

Ts. Zool. S., 1, p. 305, pl.38,f.7.

Reeve C. Syst., pl. 96, f. 1. is Chama Lazarus, Linn. 


$x^{2}+x^{3}+6$

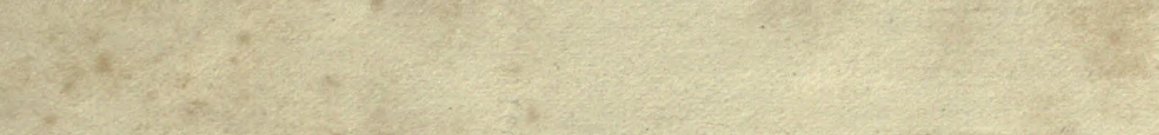

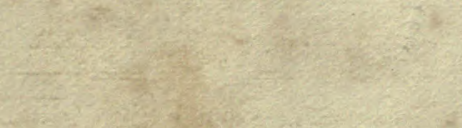

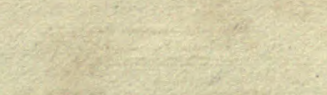

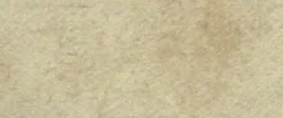

a. $x=0$

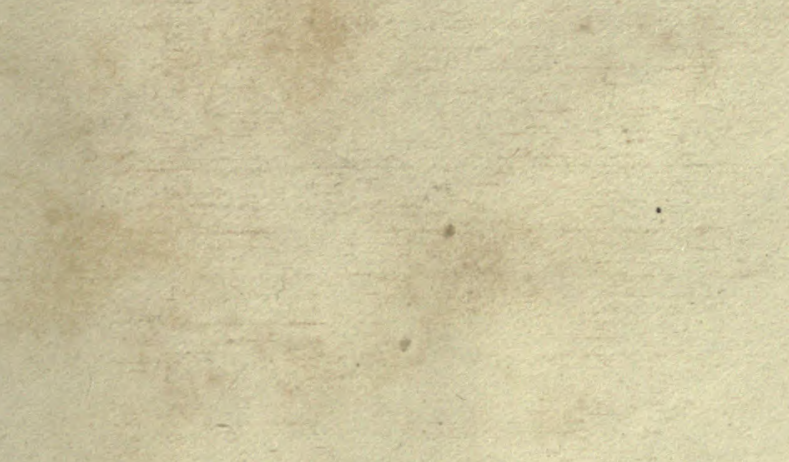

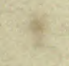
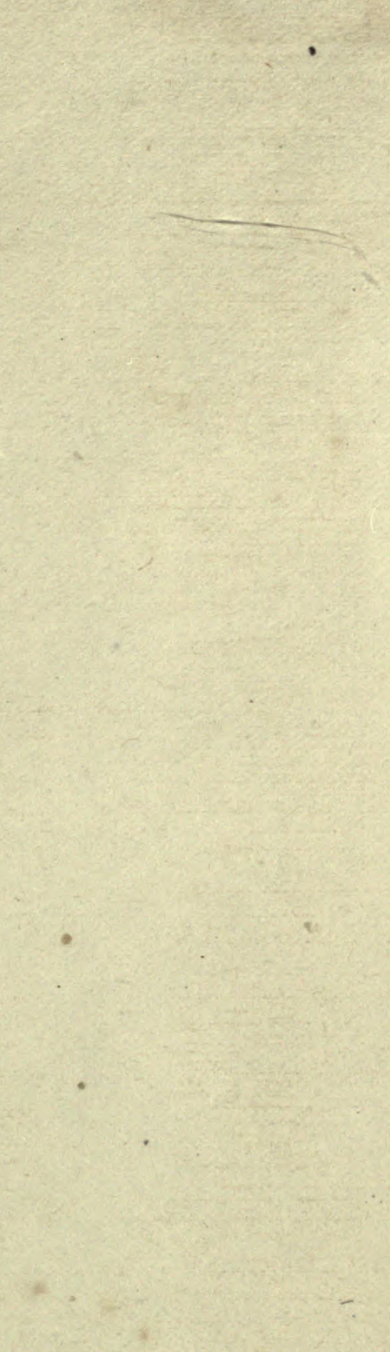


THIS BOOK IS DUE ON THE LAST DATE STAMPED BELOW

AN INITIAL FINE OF 25 CENTS WILL BE ASSESSED FOR FAILURE TO RETURN THIS BOOK ON THE DATE DUE. THE PENALTY WILL INCREASE TO 50 CENTS ON THE FOURTH DAY AND TO $\$ 1.00$ ON THE SEVENTH DAY OVERDUE.

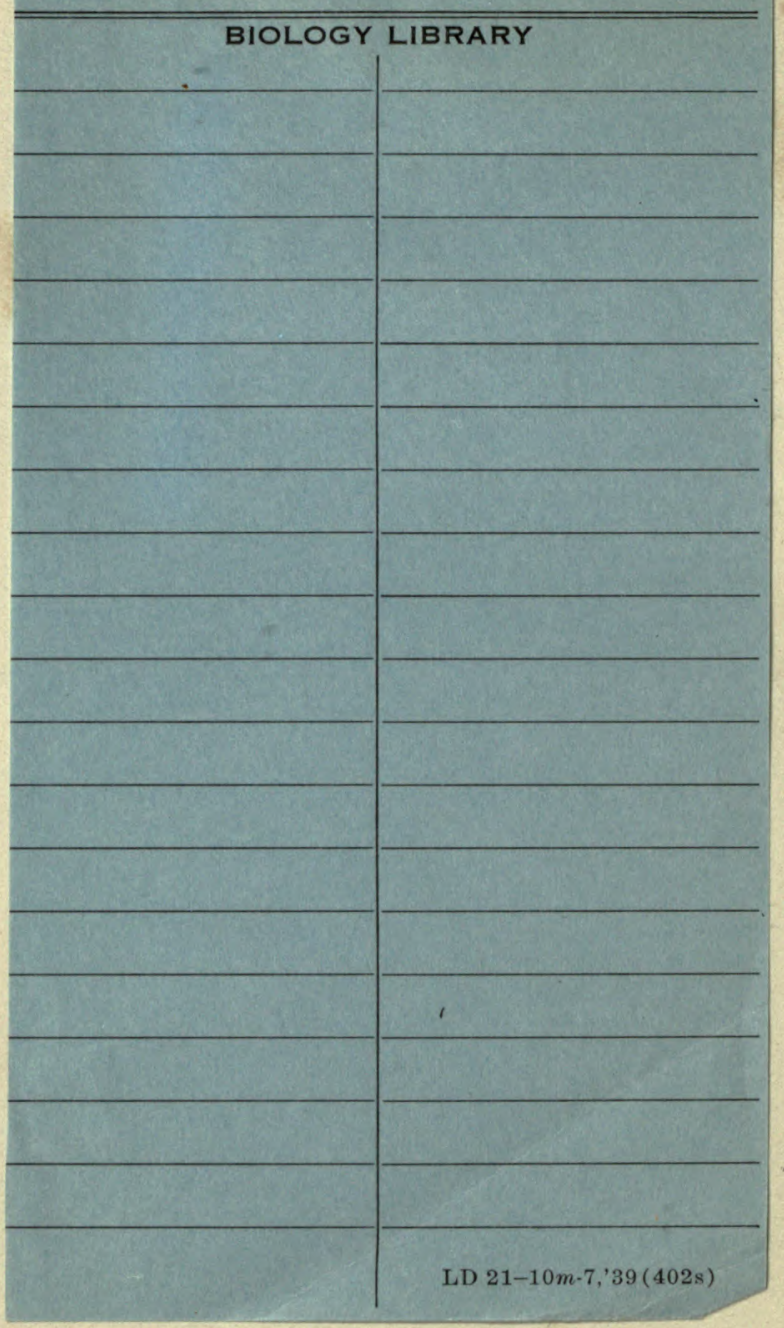


8

(4)

s

arding 3. 109

Seit

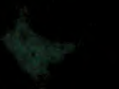

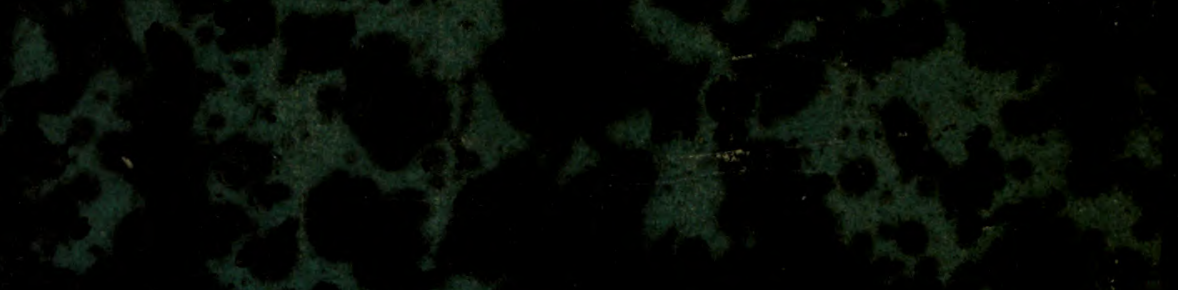

3)

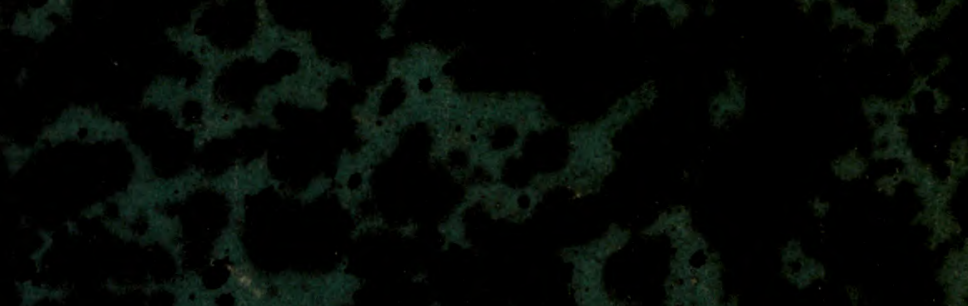

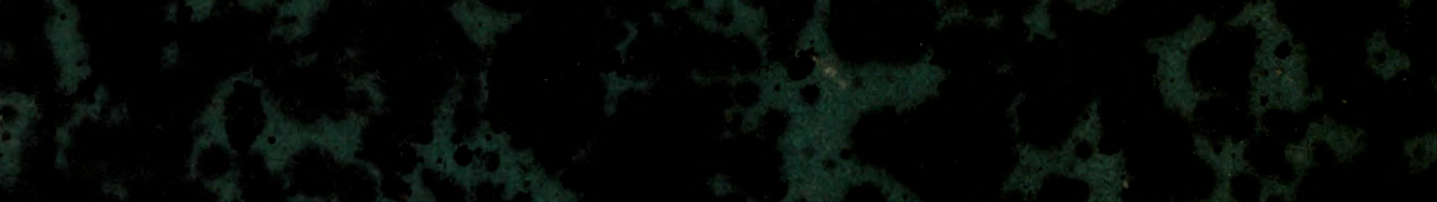

and $x^{2}+y^{5}-65$

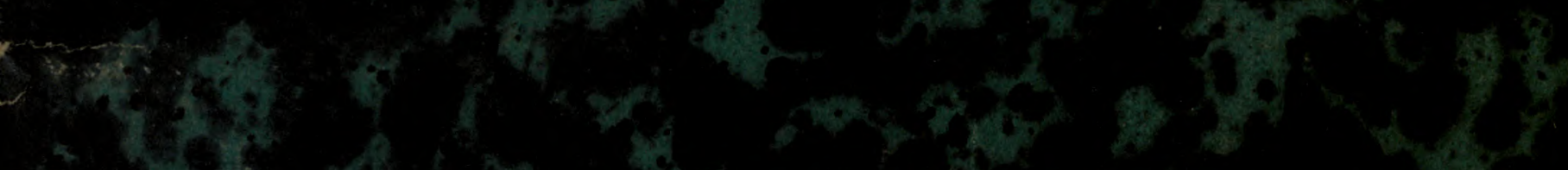

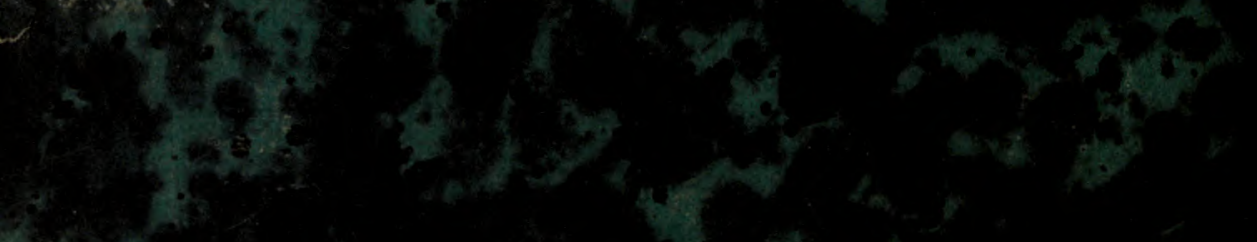

2.

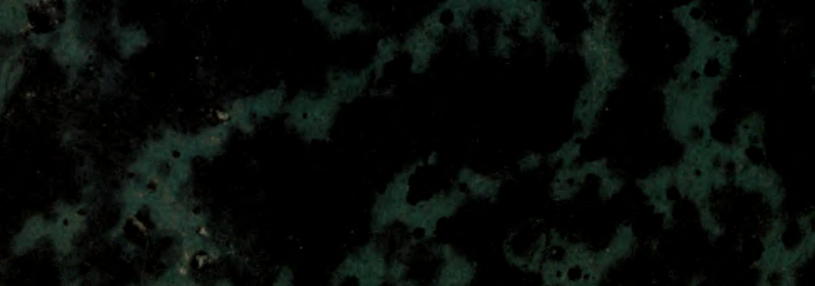

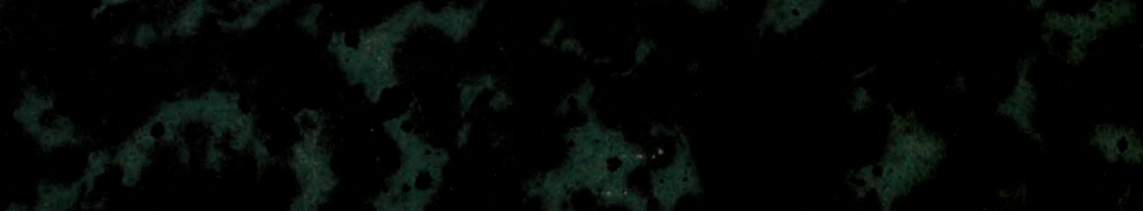

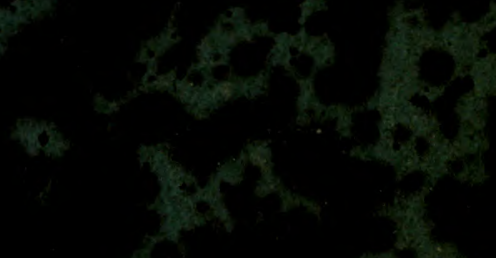

1.3.

(3)

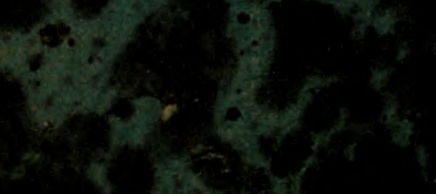

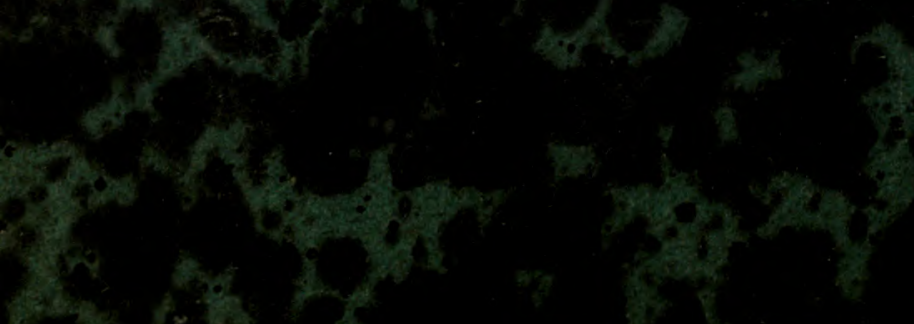

7) 5

4

s.

cots

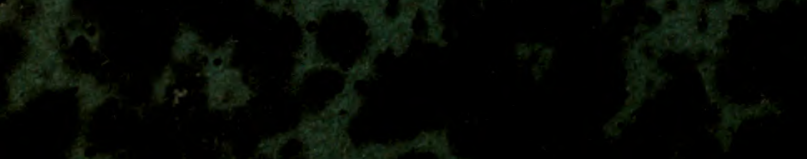

40 to

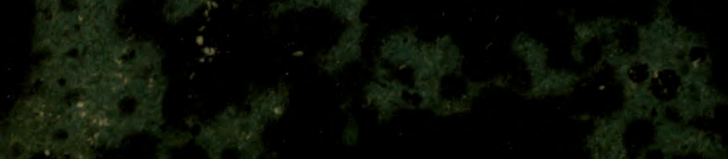

s.

4. ag at?

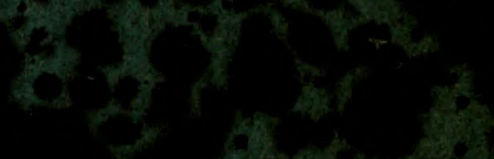

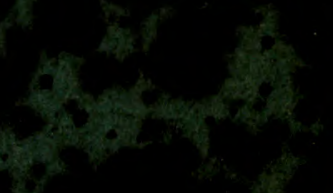

